\title{
Nanoparticle Charge and Size Control Foliar Delivery Efficiency to Plant Cells and Organelles
}

Peiguang $\mathrm{Hu}^{1 \#}$, Jing $\mathrm{An}^{2,1 \#, ~ M a q u e l a ~ M . ~ F a u l k n e r ~}{ }^{1}$, Honghong $\mathrm{Wu}^{1}$, Zhaohu $\mathrm{Li}^{2}$, Xiaoli Tian², Juan Pablo Giraldo ${ }^{1 *}$

1Department of Botany and Plant Sciences, University of California, Riverside, California 92521, USA 2State Key Laboratory of Plant Physiology and Biochemistry, College of Agronomy and Biotechnology, China Agricultural University, Beijing 100193, China

\#These authors contributed equally to this work *Corresponding author: juanpablo.giraldo@ucr.edu 
(a)

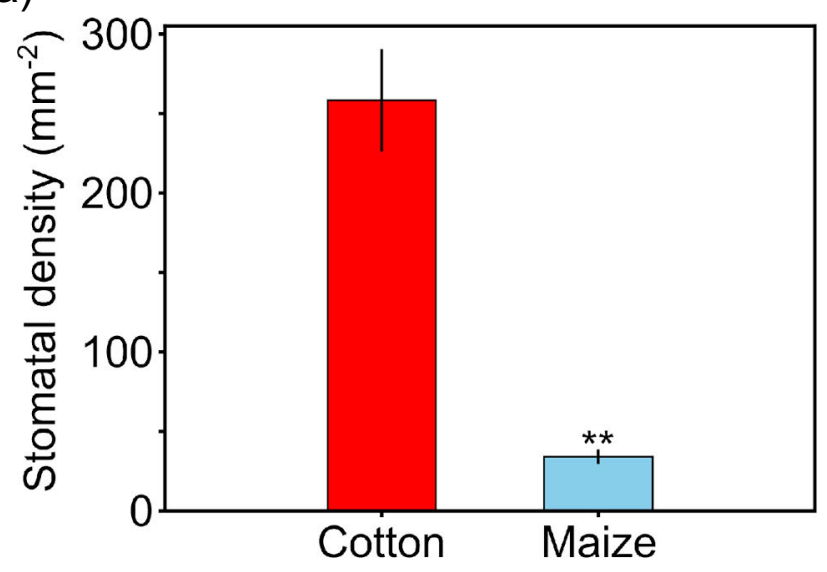

(b)

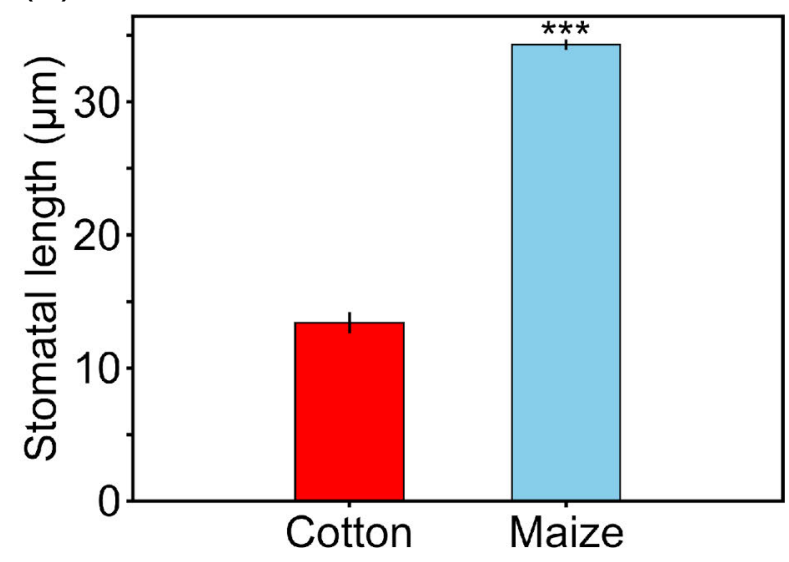

Figure S1. Stomatal density and length for cotton and maize leaves. a, Stomatal density and $\mathbf{b}$, length are shown as means $\pm \mathrm{SD}\left(\mathrm{n}=3,{ }^{* *} P<0.01\right.$, ${ }^{* * *} P<0.001$, independent samples t-test). 
(a)

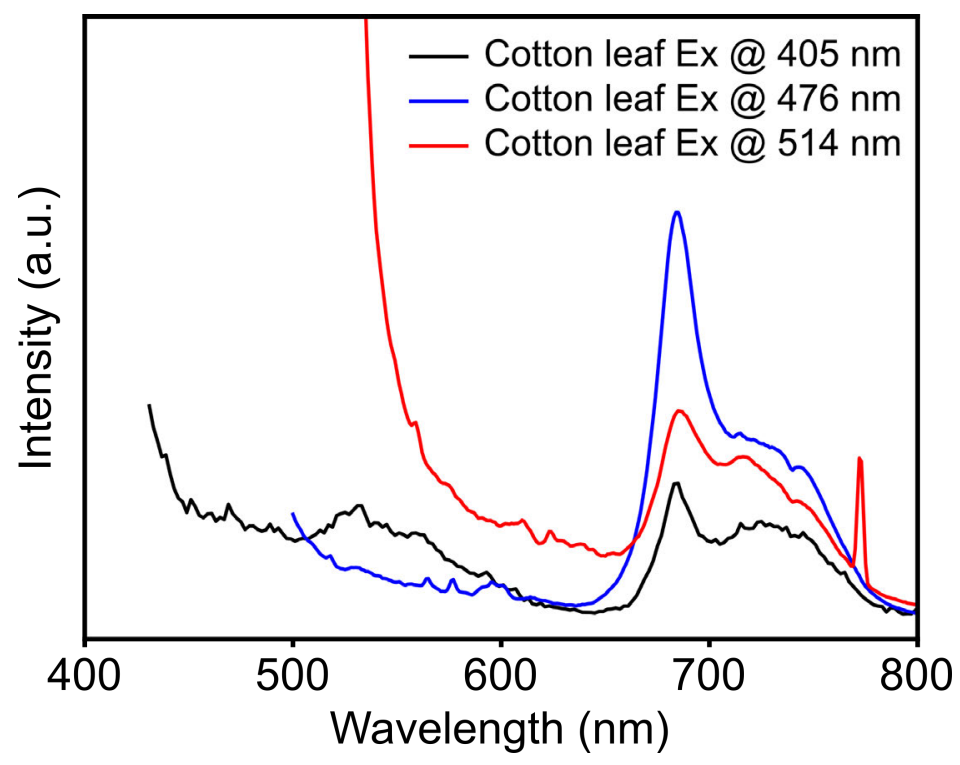

(b)

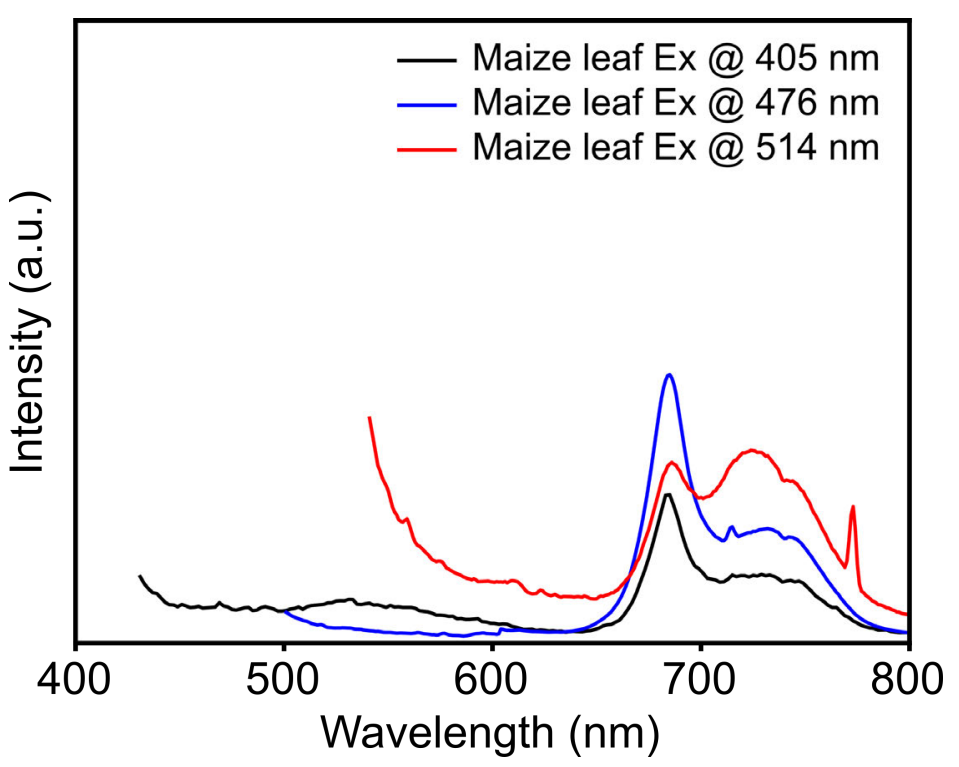

Figure S2. a, Cotton and $\mathbf{b}$, maize leaf autofluorescence emission spectra upon excitation at 405 (black), 476 (blue), and 514 (red) $\mathrm{nm}$. The same laser wavelengths were used for nanoparticle fluorescence excitation in confocal microscopy. 

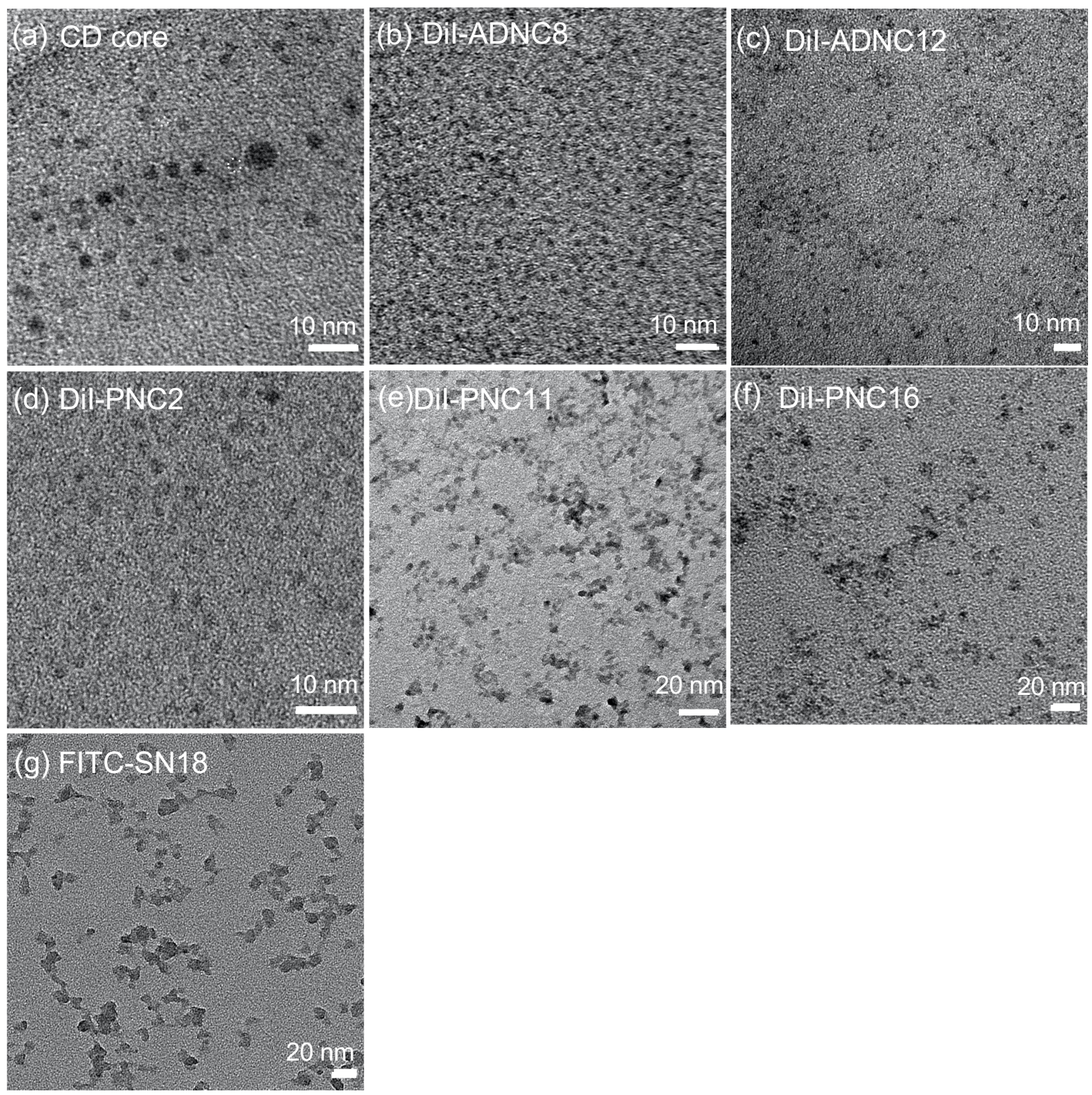

Figure S3. Representative TEM images of a, CD core, b, Dil-ADNC8, c, Dil-ADNC12, d, Dil-PNC2, e, Dil-PNC11, f, Dil-PNC16, and g, FITC-SN18. 
(a)
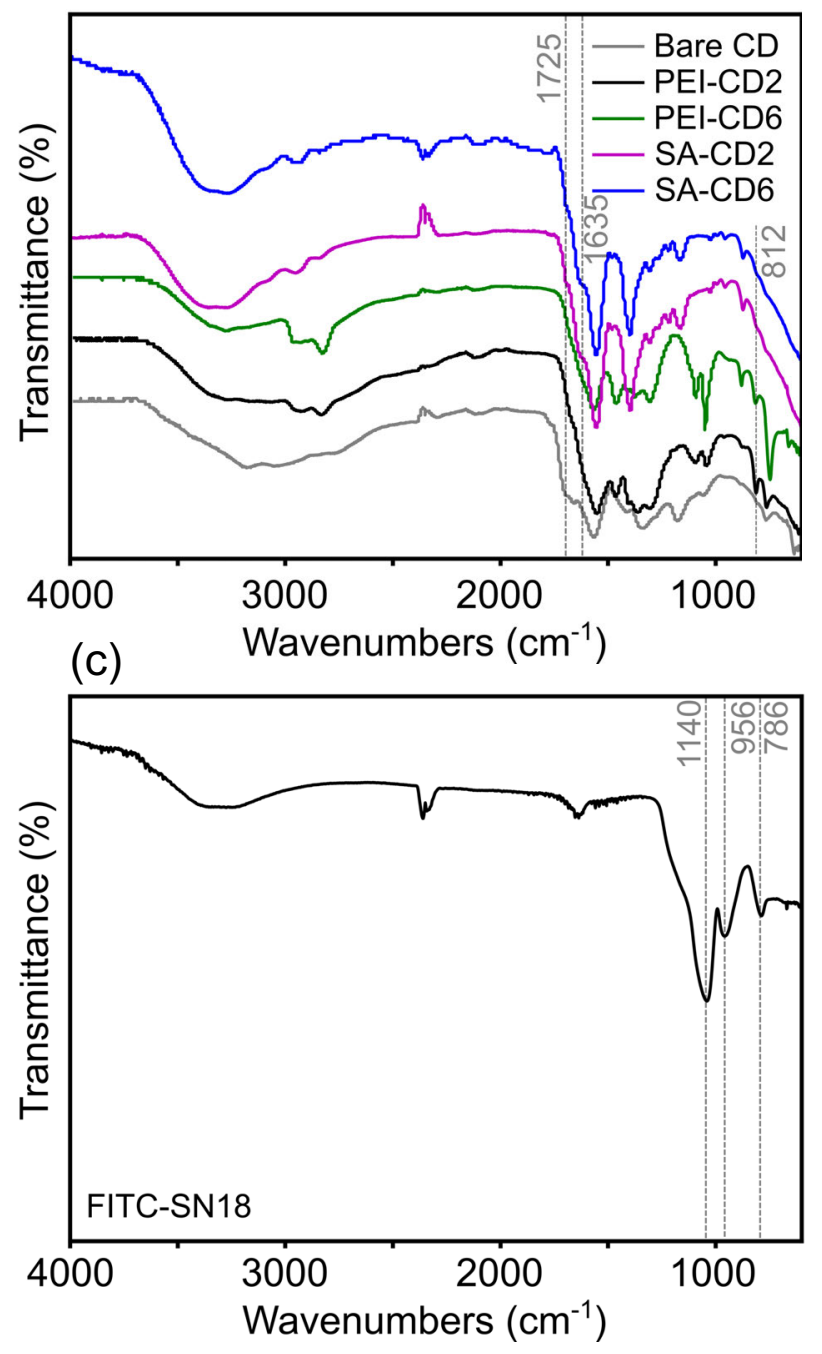

(b)

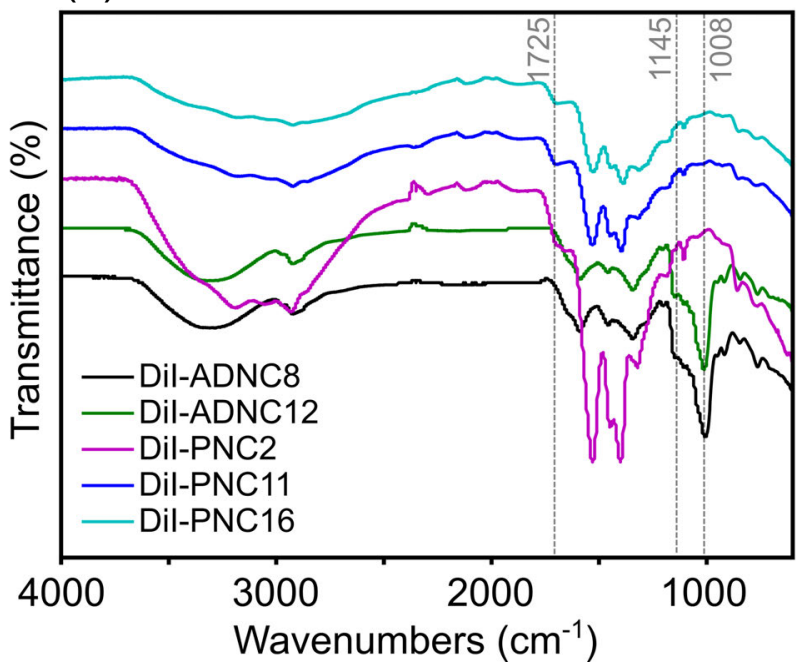

Figure S4. FTIR spectra of $\mathbf{a}, \mathrm{CDs}$, b, cerium oxide, and $\mathbf{c}$, silica nanoparticles. In comparison with bare CDs, three new FTIR peaks emerged for PEI-CDs at 1095, 1045, and $812 \mathrm{~cm}^{-1}$, corresponding to two kinds of $\mathrm{C}-\mathrm{N}$ stretching and one $\mathrm{N}-\mathrm{H}$ wagging vibrations, respectively, which indicates the successful PEI coating on the CD surface. The three FTIR peaks mentioned above were shifted or disappeared after modifying the PEI-CDs with succinic anhydride (SA) to synthesize SA-CDs. In contrast, two new shoulders were identified for SA-CDs around 1725 and $1635 \mathrm{~cm}^{-1}$ that can be assigned to different $\mathrm{C}=\mathrm{O}$ stretching vibrations in carboxyl and amide groups. These results suggest that amides were formed by the reaction of amine groups with SA and the surface of SA-CDs was functionalized with carboxyl groups. For Dil-ADNCs, two peaks were identified at 1145 and $1008 \mathrm{~cm}^{-1}$ that can be ascribed to the $\mathrm{C}-\mathrm{N}$ stretching and $\mathrm{C}-\mathrm{O}-\mathrm{C}$ stretching vibrations, indicating that the dextran was successfully coated on $\mathrm{CeO}_{2} \mathrm{NPs}$ and further functionalized with DEAE (diethylaminoethyl). In contrast, all Dil-PNCs exhibited a peak at $1725 \mathrm{~cm}^{-1}$, which can be attributed to the $\mathrm{C}=\mathrm{O}$ stretching vibration of carboxyl groups, meaning the successful PAA (poly(acrylic acid)) coating on $\mathrm{CeO}_{2}$ NP surface. The FITC-SN18 exhibit three characteristic peaks at 1140,956 , and $786 \mathrm{~cm}^{-1}$, which can be ascribed to the vibration of $\mathrm{Si}-\mathrm{O}-\mathrm{Si}, \mathrm{Si}-\mathrm{OH}$, and $\mathrm{Si}-\mathrm{O}-\mathrm{Si}$ bonds, respectively. This indicates the successful formation of silica nanoparticles with silanol groups on the surface. No characteristic vibration bands were identified for covalently incorporated FITC molecules, which might be due to the low loading of the fluorophore dye. 
(a)



(c)

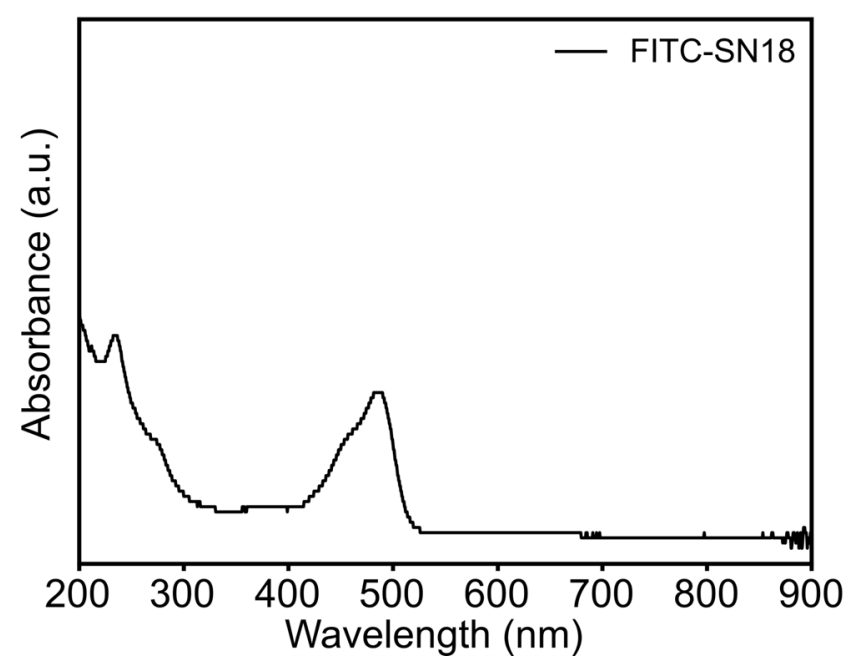

(b)

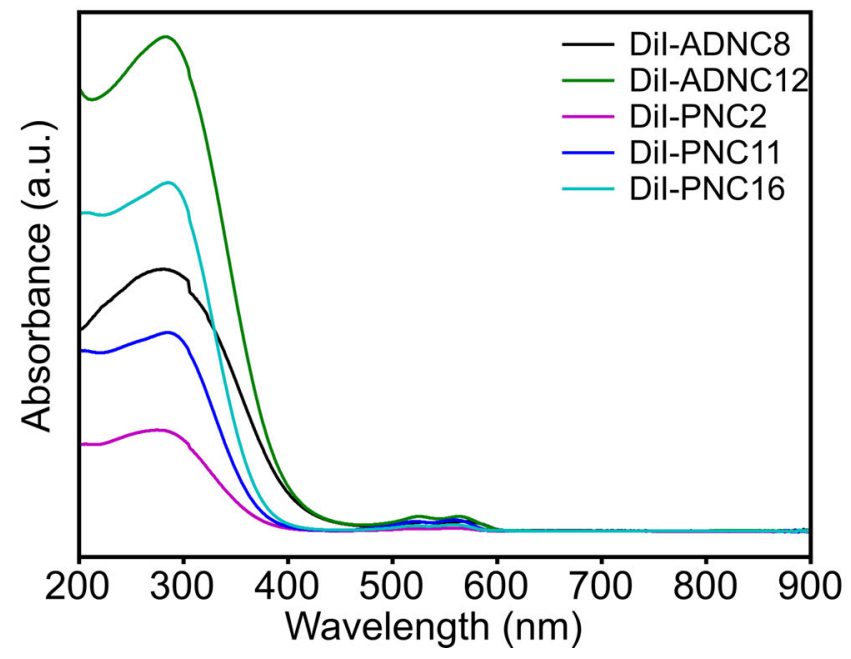

Figure S5. UV-vis absorption spectra of $\mathbf{a}, \mathrm{CDs}$, b, cerium oxide, and $\mathbf{c}$, silica nanoparticles. All PEI-CDs and SA-CDs exhibited an absorption shoulder at around 270 $\mathrm{nm}$ and an absorption peak at around $325 \mathrm{~nm}$, which can be assigned to the $\pi-\pi^{*}$ transition of the aromatic domains in carbonic core and the $n-\pi^{*}$ transition of conjugated $\mathrm{C}=\mathrm{O}$ bonds on the surface, respectively. The Dil-PNCs and Dil-ADNCs have a strong absorption peak within the range from 270 to $290 \mathrm{~nm}$. The other two absorption peaks at 525 and $570 \mathrm{~nm}$ can be assigned to Dil, confirming successful encapsulation of the dye in the coating polymers. One broad absorption band with maximum at $485 \mathrm{~nm}$ was identified for the FITC-SN18, indicating the successful labeling by the fluorophore dye of FITC. 

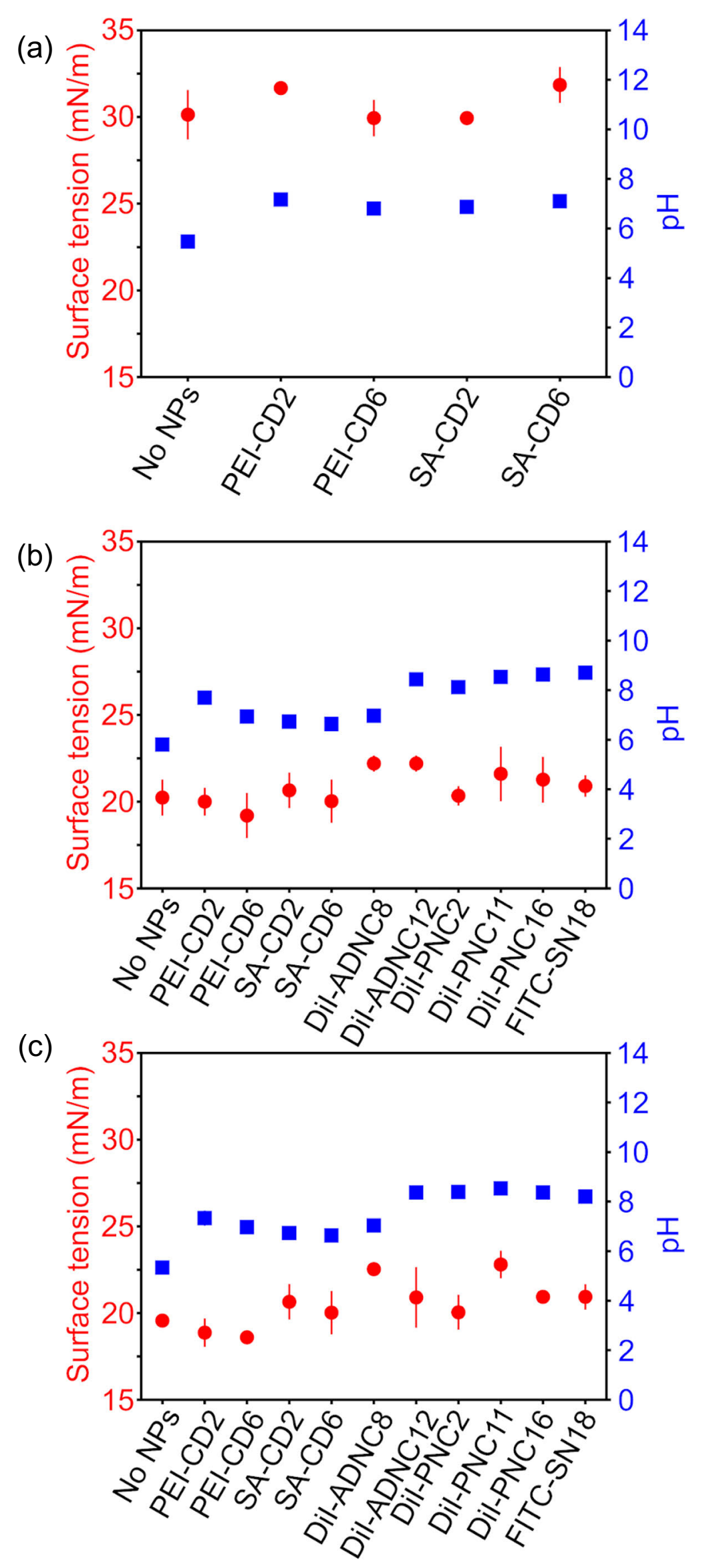

Figure S6. Surface tension (red) and $\mathrm{pH}$ (blue) of different foliar nanoparticle formulations made with a, Triton X-100 (0.2\%), b, Silwet L-77 (0.2\%), and c, Silwet L-77 (0.3\%) and glycerol (3\%). Mean \pm SD. 


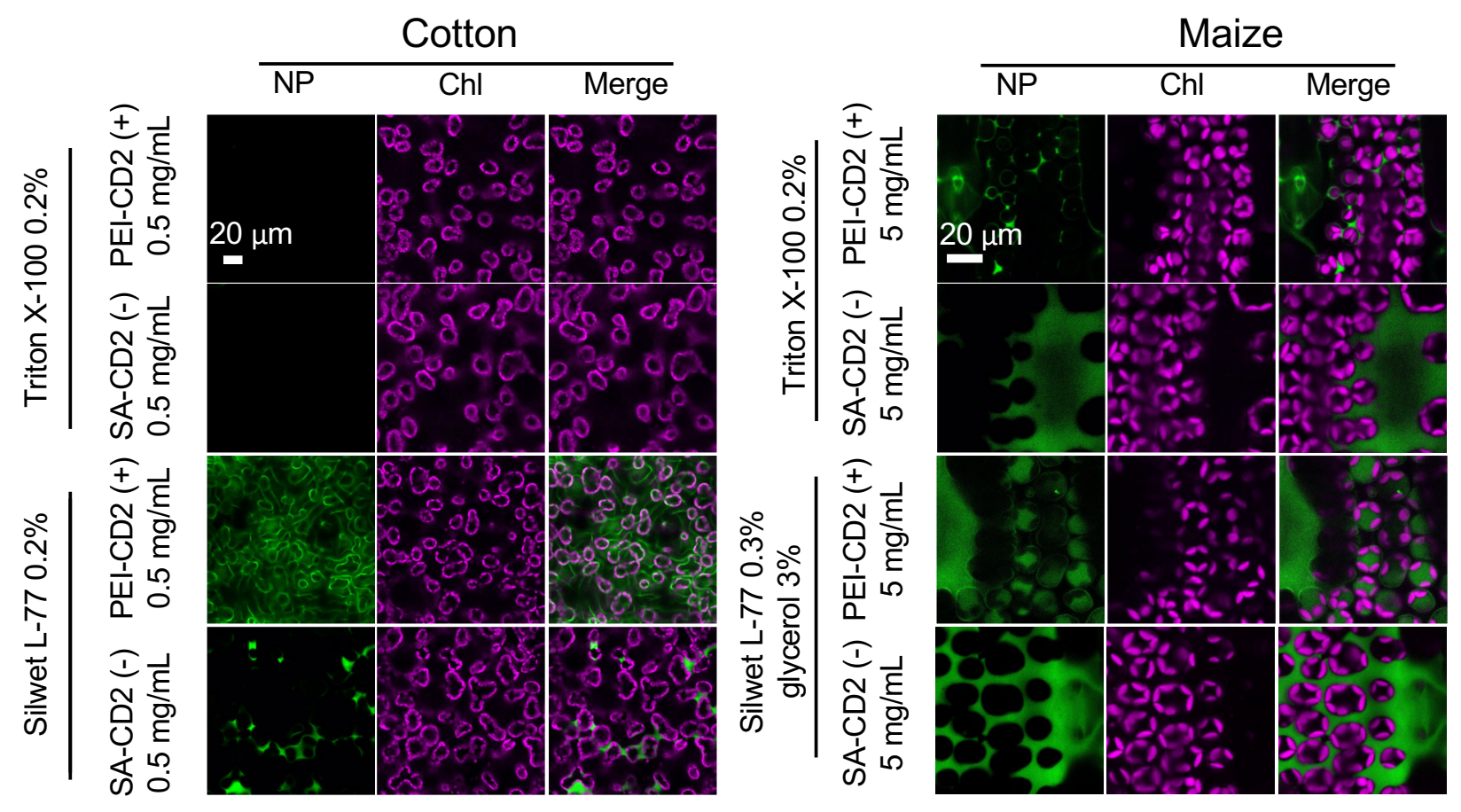

Figure S7. Representative confocal microscopy images of leaf mesophyll cells after foliar delivery of nanoparticles suspended in formulations with Triton X-100 or Silwet L-77 as surfactant. Nanoparticles (PEI-CD2 and SACD2) can be delivered to leaf mesophyll cells containing chloroplasts by using either Triton X-100 or Silwet L-77 as surfactant. NP and Chl represent nanoparticles (green) and chloroplasts (magenta). The $(+)$ indicates positively charged nanoparticles. 

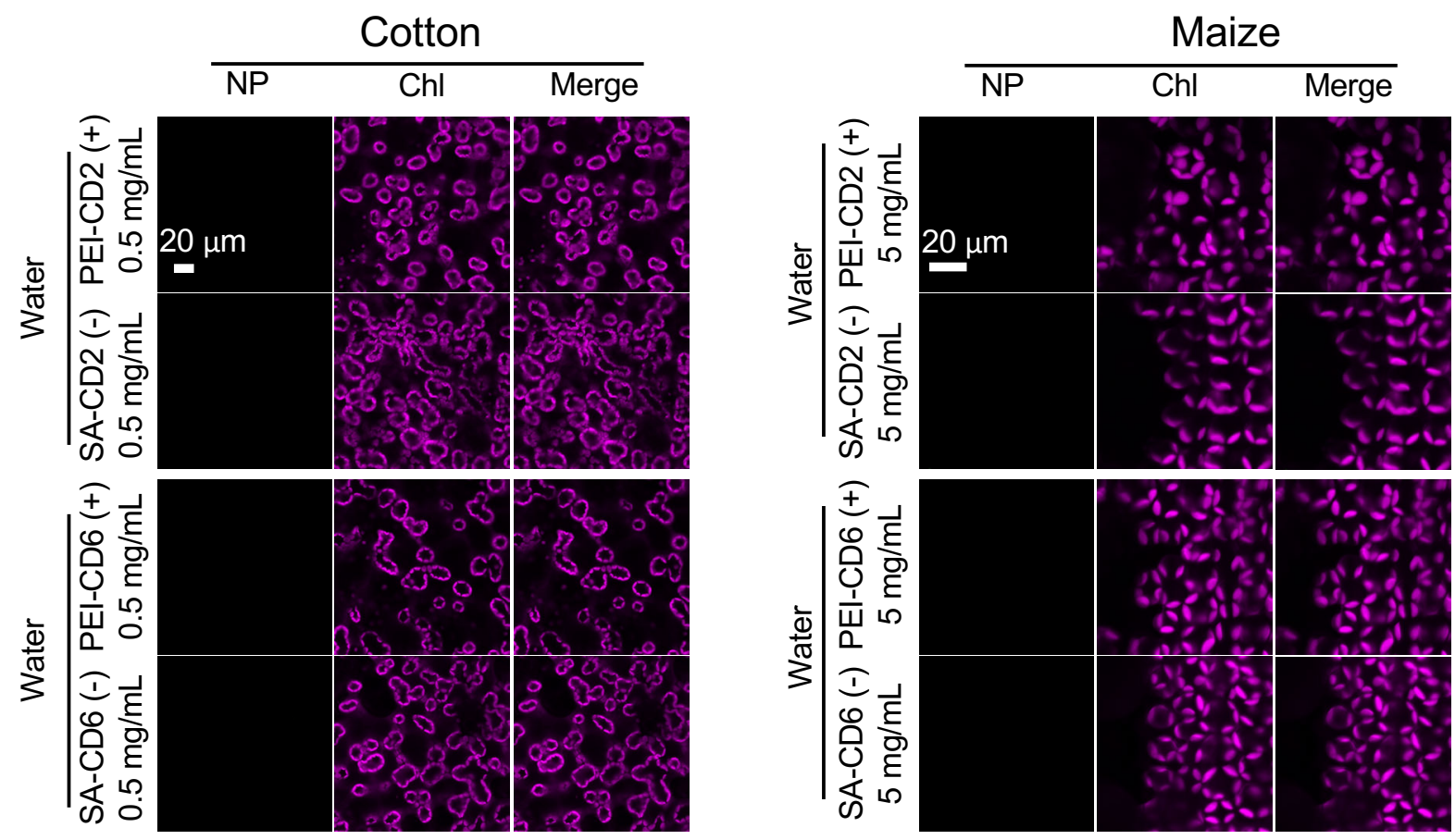

Figure S8. Representative confocal microscopy images of cotton and maize leaf mesophyll after foliar delivery of nanoparticles suspended in water without surfactant. No nanoparticles were observed in both cotton (left) and maize (right) leaves. Chl represents Chloroplasts (magenta). The (+) indicates positively charged nanoparticles. 
(a)

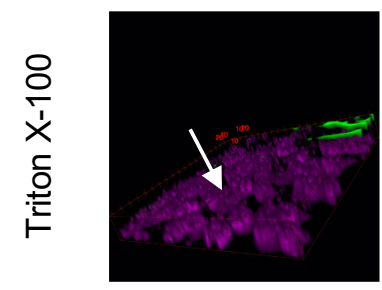

(+) PEI-CD2

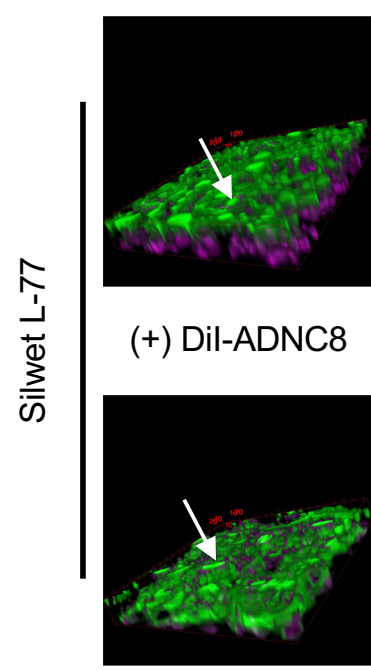

(b)

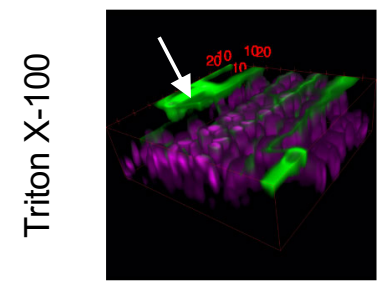

(+) PEI-CD2

$\stackrel{\stackrel{N}{N}}{\sum}$
(-) SA-CD2

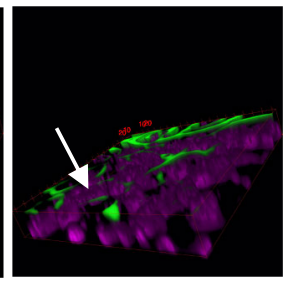

(-) SA-CD2

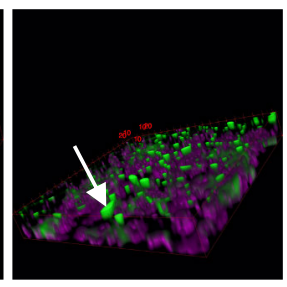

(-) Dil-PNC11

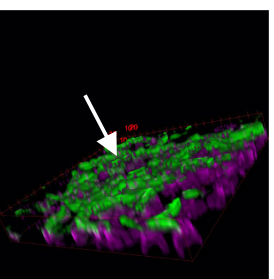

(-) SA-CD2

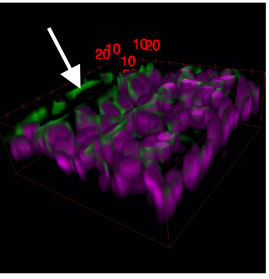

$(-)$ SA-CD2

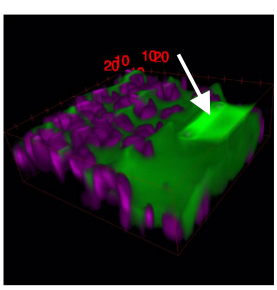

(-) Dil-PNC11

(+) Dil-ADNC8
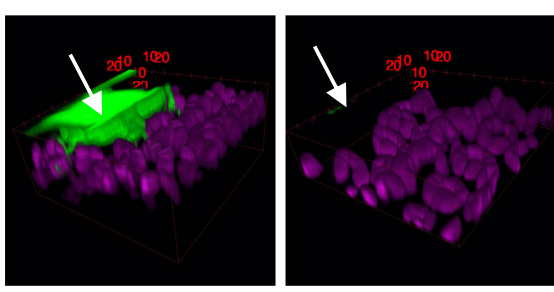

(+) PEI-CD6

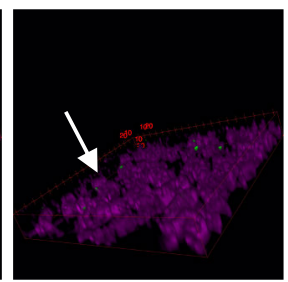

(-) Dil-PNC2

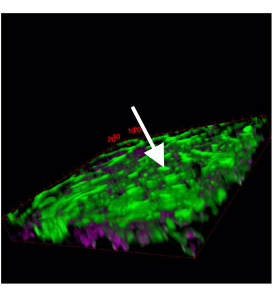

(+) Dil-ADNC12

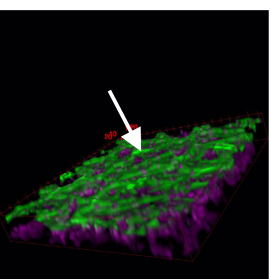

(+) PEI-CD6

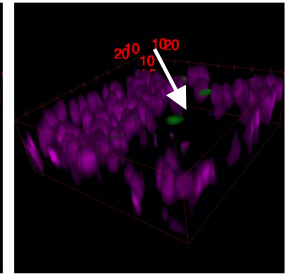

(-) Dil-PNC2

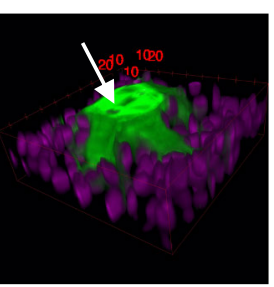

(+) Dil-ADNC12

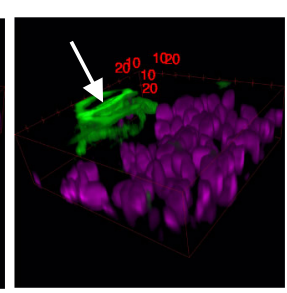

(-) SA-CD6

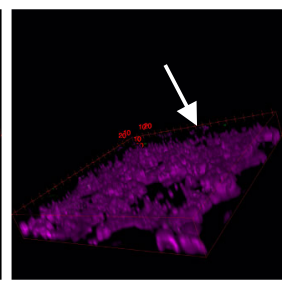

(+) PEI-CD6

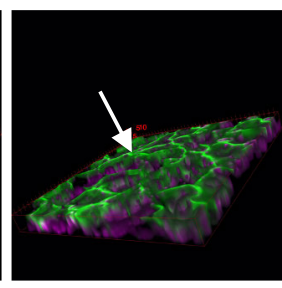

(-) Dil-PNC16

(-) FITC-SN18

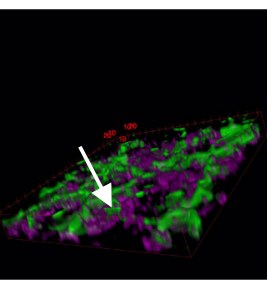

(-) SA-CD6


(+) PEI-CD6

(-) SA-CD6

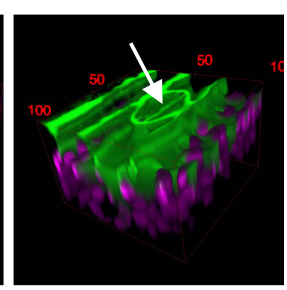

(-) Dil-PNC16



(-) FITC-SN18
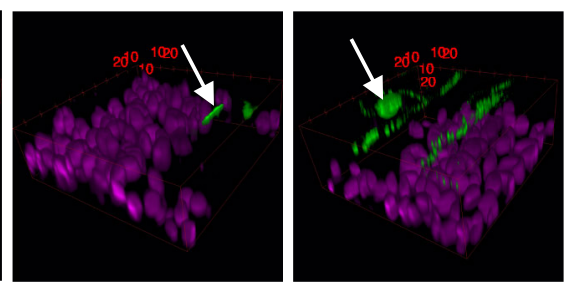

Figure S9. 3D renderings of confocal microscopy images showing nanoparticle delivery pathways in $\mathbf{a}$, cotton and $\mathbf{b}$, maize, from the leaf surface into mesophyll cells. Green and magenta represent nanoparticles and chloroplast autofluorescence, respectively. Stomatal pores are indicated by white arrows. The (+) and (-) indicate positively and negatively charged nanoparticles, respectively. 

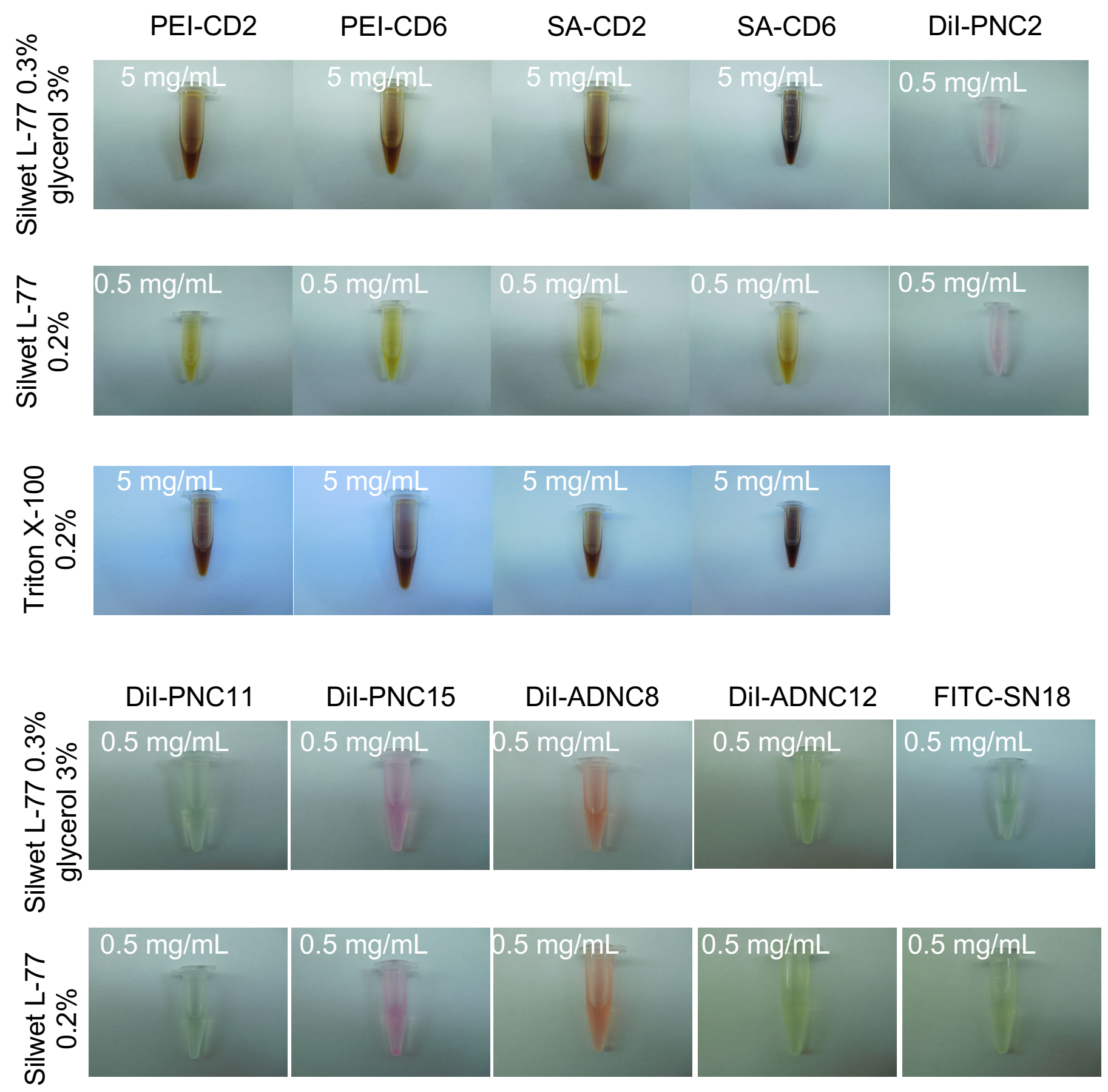

Figure S10. Nanoparticle high stability in surfactant formulation was assessed by centrifugation. Images of nanoparticle formulations after centrifugation (13.2k rpm) for 15 min showed no precipitate on the bottom of the Eppendorf tubes. 
(a)

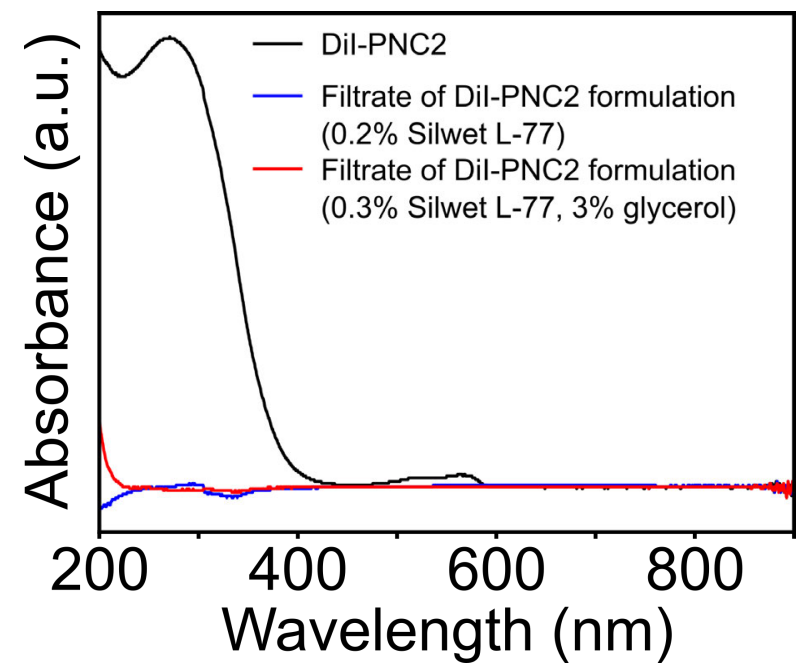

(b)

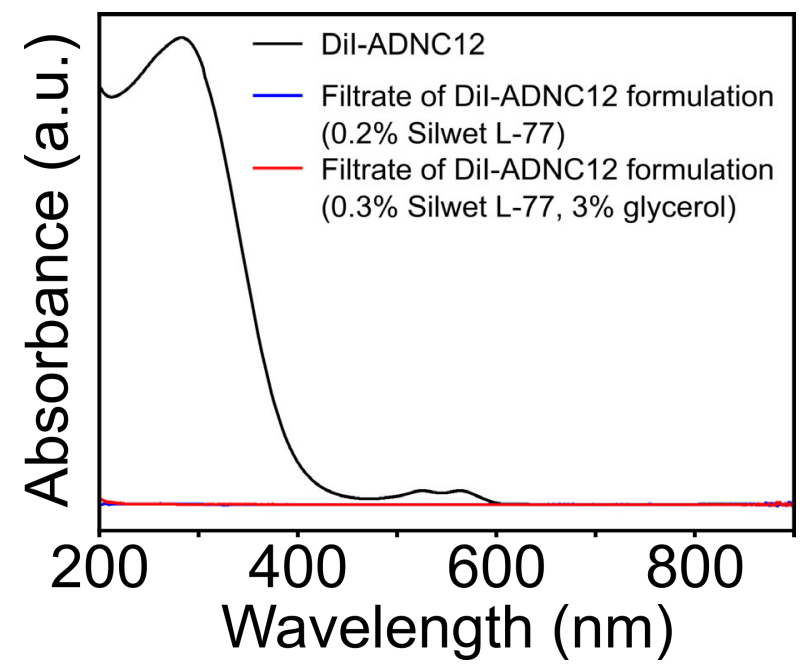

(c)

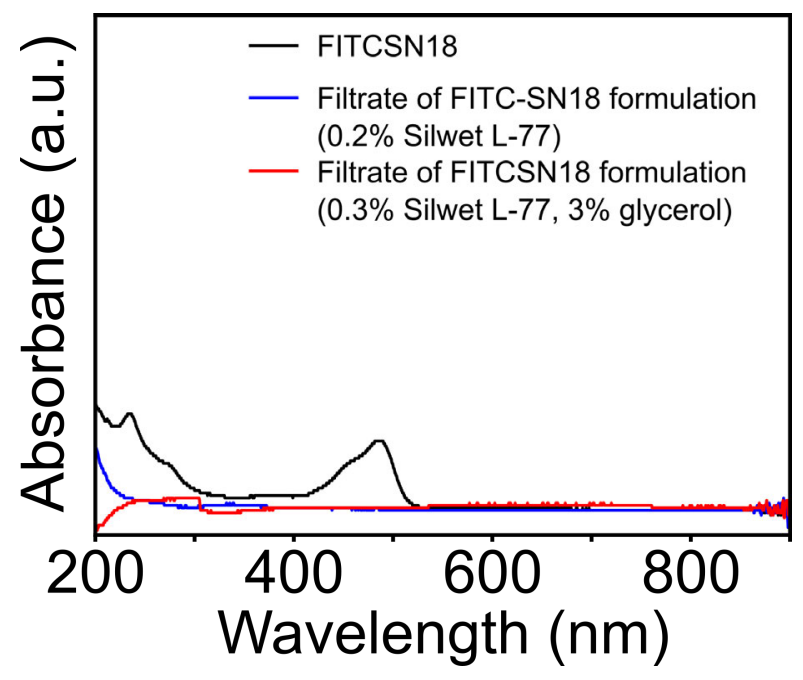

Figure S11. UV-vis absorption spectra of nanoparticles labeled with fluorescent dyes, a, Dil-PNC2, b, Dil-ADNC12, and c, silica nanoparticles and filtrate collected after centrifugation (4500 rpm, Amicon cell, MWCO 3k, Millipore Inc.) in Silwet L-77. The filtrate of Dil-PNC2 and Dil-ADNC12 shows no absorbance within the range from 450 to $650 \mathrm{~nm}$ where Dil exhibits strong absorbance. This confirms no Dil molecules leak from the coating polymers (PAA or dextran) even in the presence of surfactant (Silwet L-77). The filtrate of FITC-SN18 shows no absorbance within the range from 400 to $520 \mathrm{~nm}$ where FITC exhibits strong absorbance. Therefore, no FITC molecules leak from the Silica nanoparticles in Silwet L-77. 



Figure S12. Chlorophyll content patterns in cotton and maize leaves after exposure to foliar nanoparticle formulations containing CDs in Silwet L-77 as surfactant. 
Cotton

PEI-CD6 with Silwet L-77 0.2\%

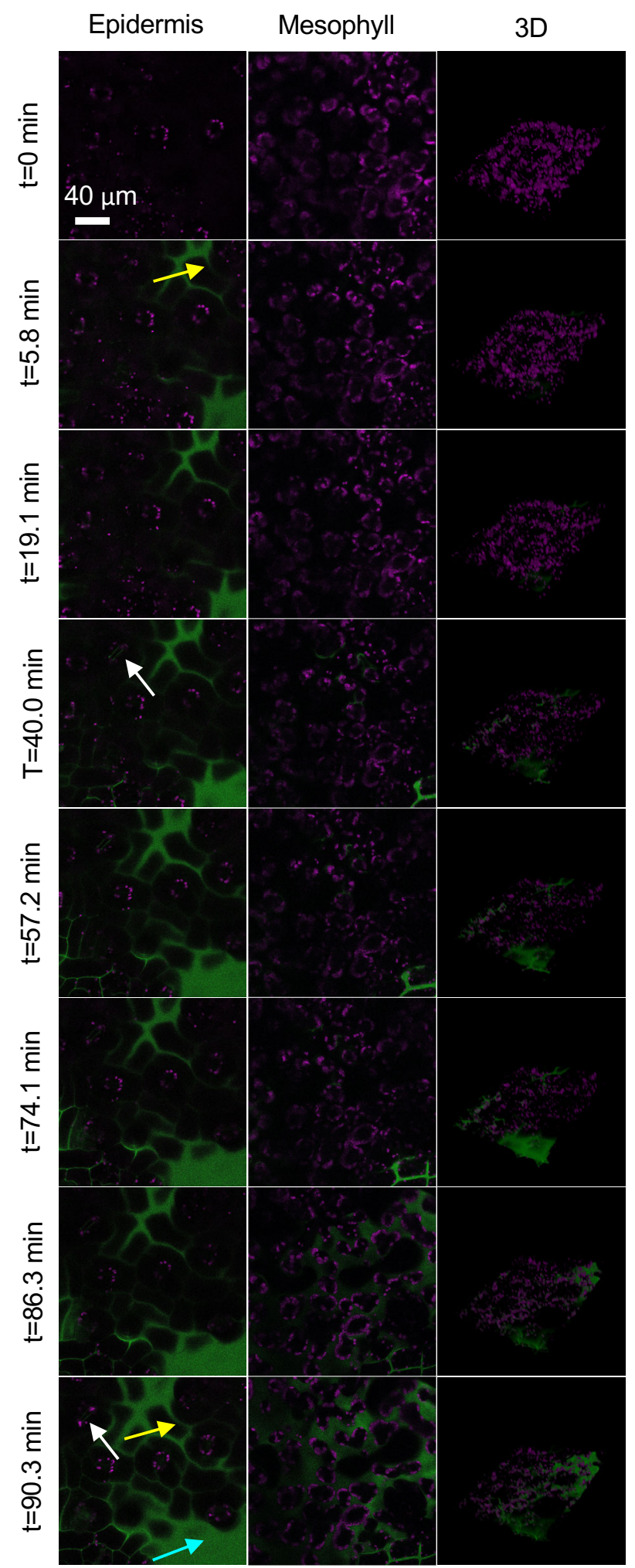

Maize

PEI-CD6 with Silwet L-77 0.3\%

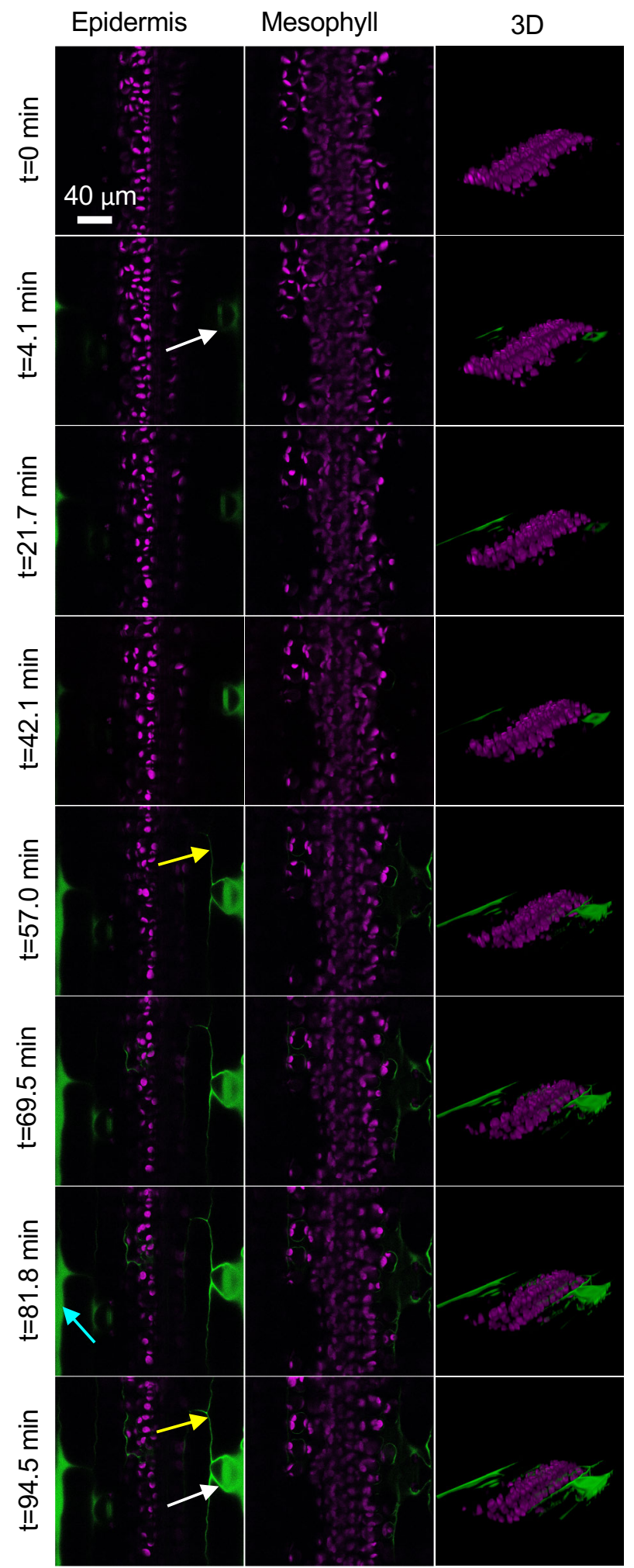

Figure S13. Snapshots from confocal fluorescence microscopy videos showing PEICD6 (green) translocation in real-time from the leaf epidermis into mesophyll cells and chloroplasts (magenta). PEI-CD6 delivered in Silwet L-77 move rapidly through both stomatal and cuticular pathways for cotton, whereas mainly through stomatal pathway in maize. The delivery into both cotton and maize leaves occurred within 30-40 min of nanoparticle exposure. White and yellow arrows point to stomatal and cuticular pathways, respectively. Cyan arrows point to areas flooded with CDs due to uneven leaf surface. $\mathrm{t}=0 \mathrm{~min}$ represents images captured before nanoparticle formulation was added. 
Cotton

SA-CD2 with Silwet L-77 0.2\%

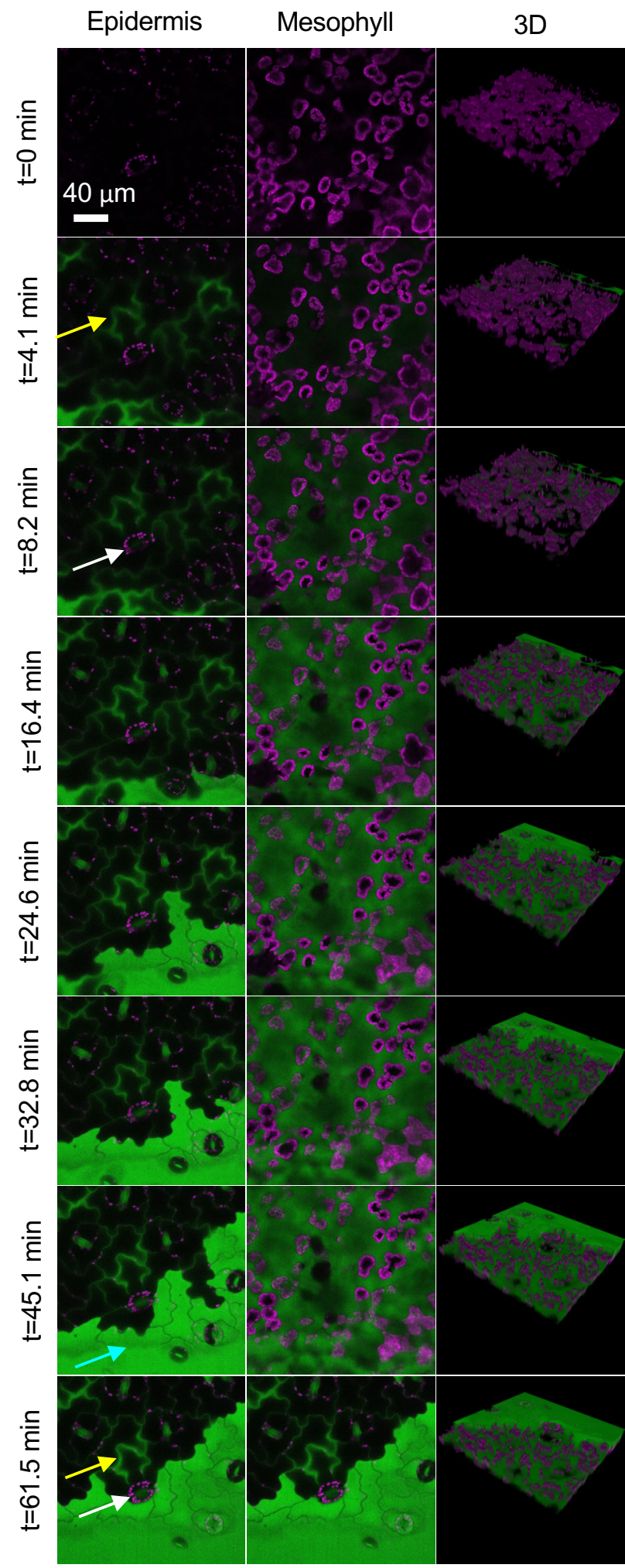

Maize

SA-CD2 with Silwet L-77 0.3\%

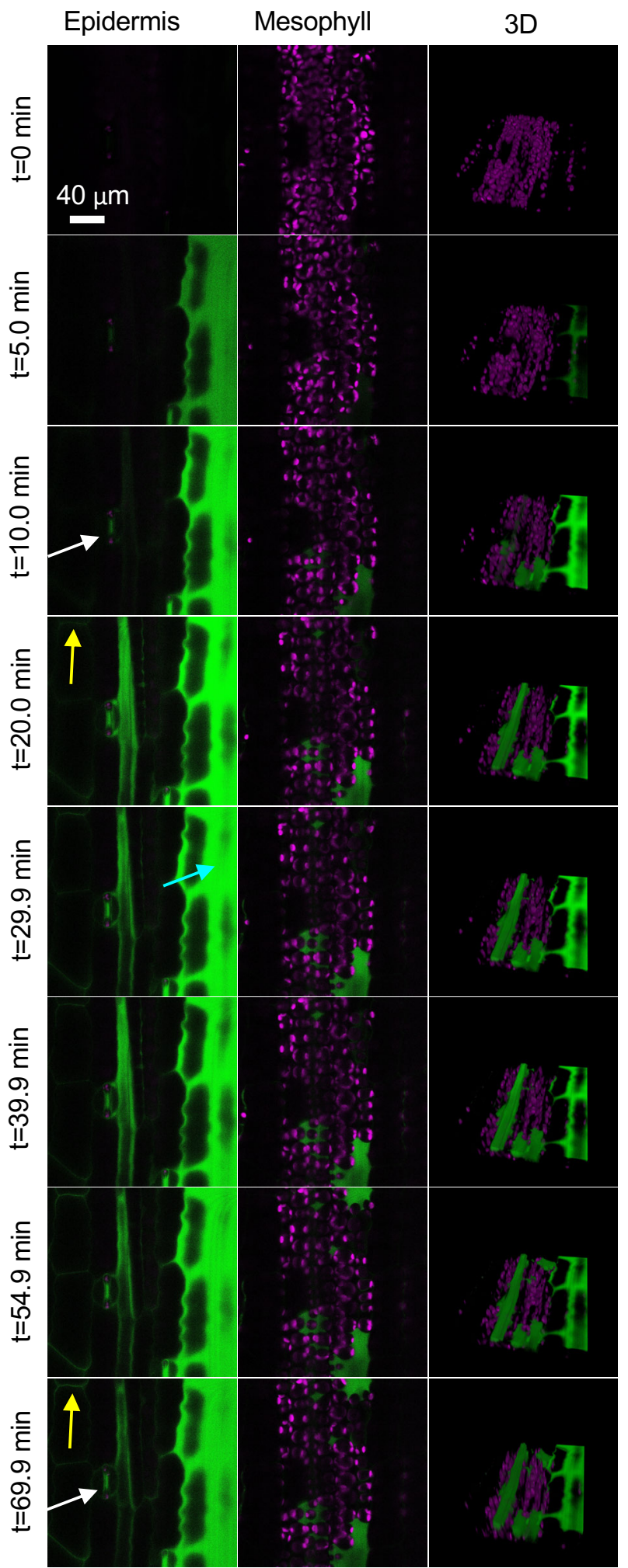

Figure S14. Snapshots from confocal fluorescence microscopy videos showing SACD2 (green) translocation in real-time from the leaf epidermis into mesophyll cells and chloroplasts (magenta). SA-CD2 delivered in Silwet L-77 move rapidly through both stomatal and cuticular pathways for cotton, whereas mainly through stomatal pathway in maize. The delivery into both cotton and maize leaves occurred within 10-20 min of nanoparticle exposure. White and yellow arrows point to stomatal and cuticular pathways, respectively. Cyan arrows point to areas flooded with CDs due to uneven leaf surface. $\mathrm{t}=0$ min represents images captured before nanoparticle formulation was added. 
Cotton

SA-CD6 with Silwet L-77 0.2\%

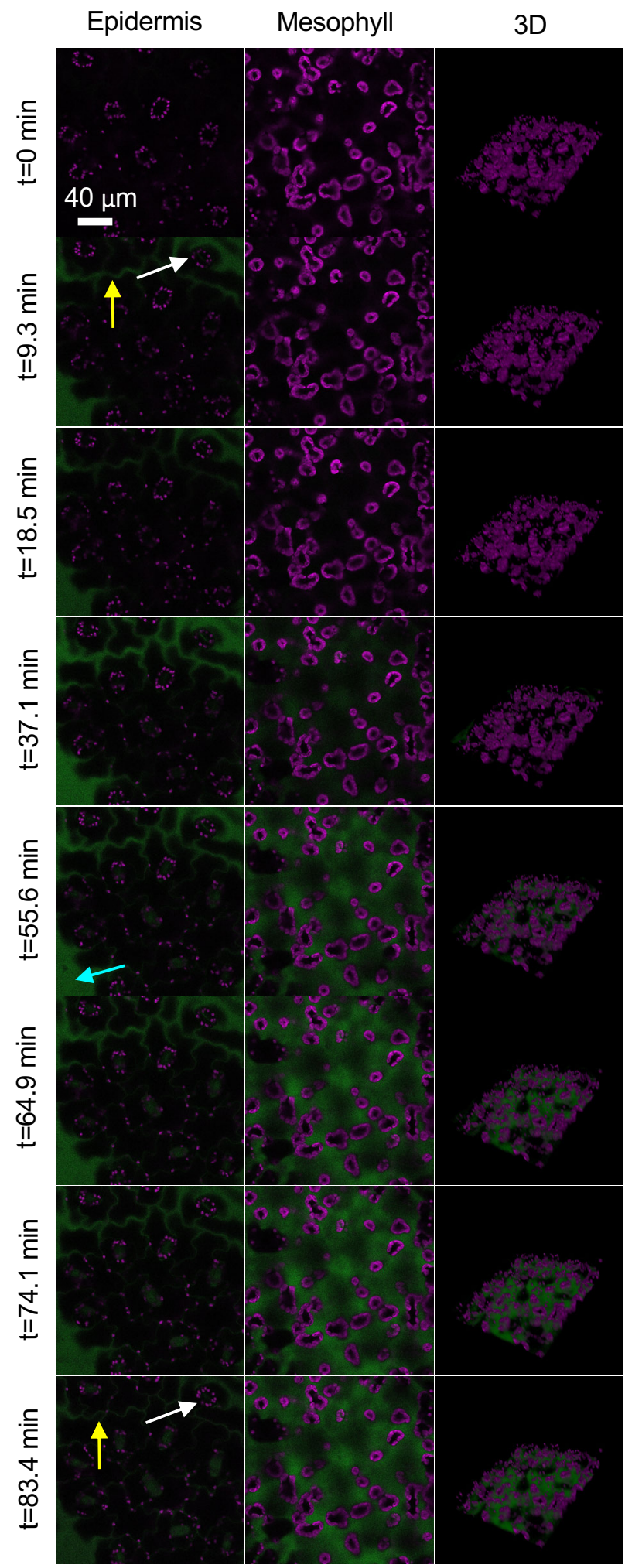

Maize

SA-CD6 with Silwet L-77 0.3\%

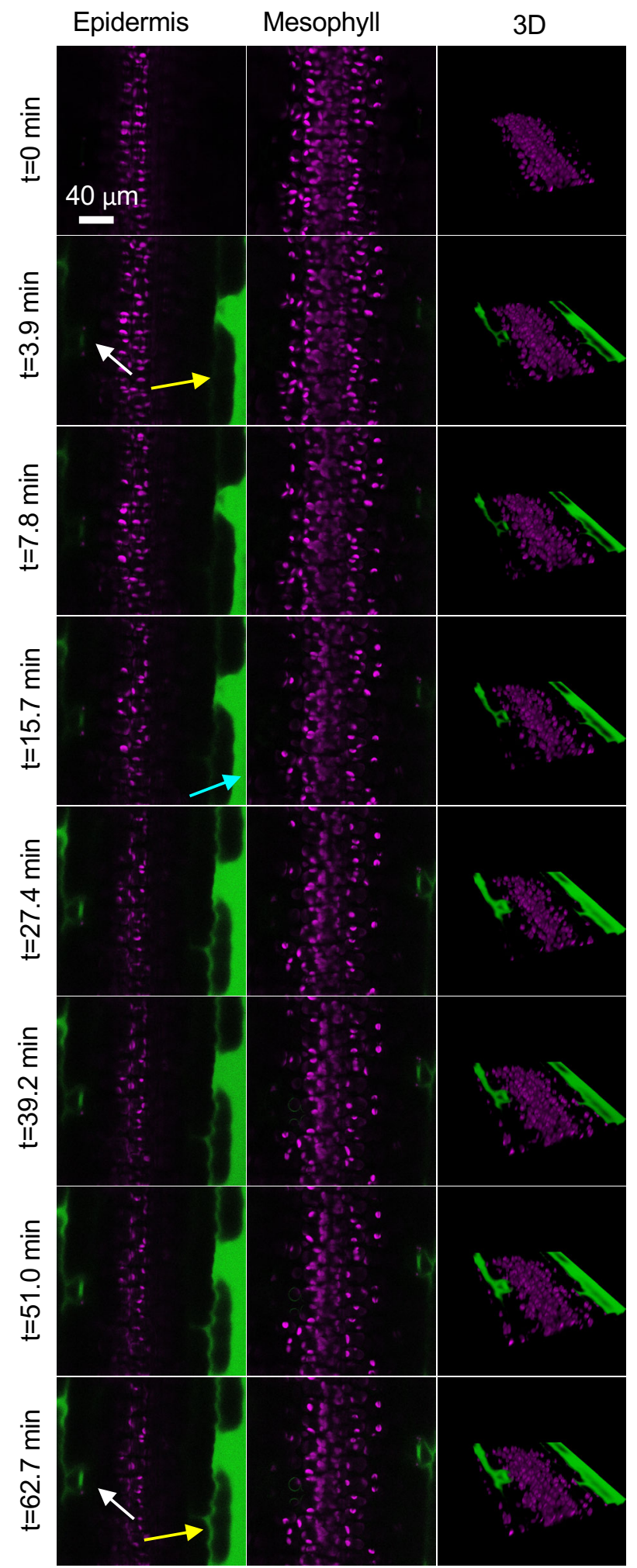

Figure S15. Snapshots from confocal fluorescence microscopy videos showing SACD6 (green) translocation in real-time from the leaf epidermis into mesophyll cells and chloroplasts (magenta). SA-CD6 delivered in Silwet L-77 move rapidly through both stomatal and cuticular pathways for cotton, whereas only stomatal pathway in maize. The delivery into both cotton and maize leaves occurred within 30-40 min of nanoparticle exposure. White and yellow arrows point to stomatal and cuticular pathways, respectively. Cyan arrows point to areas flooded with CDs due to uneven leaf surface. $\mathrm{t}=0$ min represents images captured before nanoparticle formulation was added. 


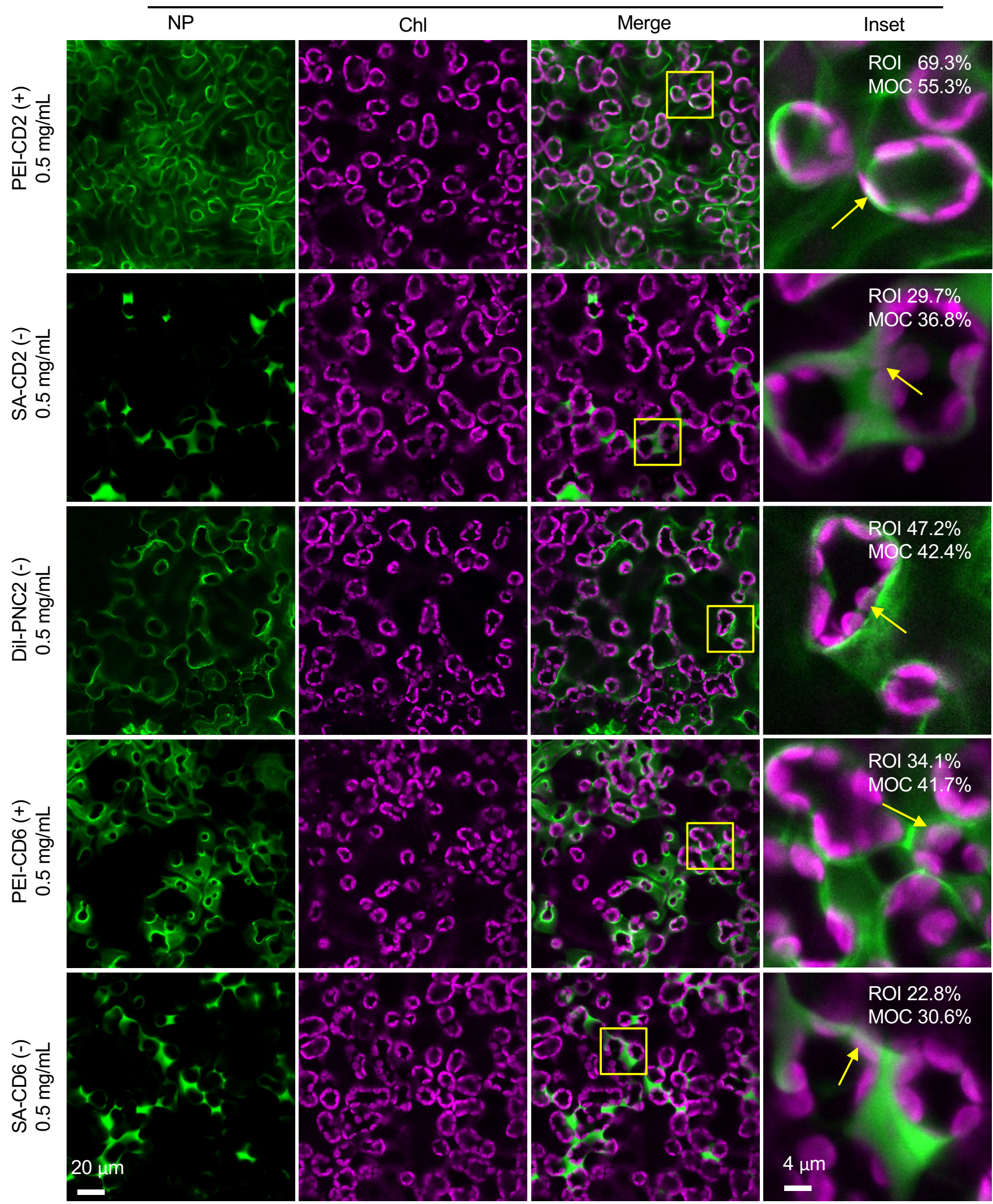

Figure S16. Confocal microscopy images of cotton leaf mesophyll cells with chloroplasts after foliar delivery of nanoparticles suspended in Silwet L-77. Inset values are colocalization rates based on ROI method and Manders' overlap coefficients (MOC), representing the fraction of chloroplast pixels overlapping with nanoparticle pixels. Yellow arrows indicate examples of colocalization of nanoparticles (NP, green) and chloroplasts (Chl, magenta), respectively. The (+) and (-) indicate positively and negatively charged nanoparticles, respectively. 




Figure S17. Confocal microscopy images of cotton leaf mesophyll cells with chloroplasts after foliar delivery of nanoparticles suspended in Silwet L-77. Inset values are colocalization rates based on ROI method and Manders' overlap coefficients (MOC), representing the fraction of chloroplast pixels overlapping with nanoparticle pixels. Yellow arrows indicate examples of colocalization of nanoparticles (NP, green) and chloroplasts (Chl, magenta), respectively. The $(+)$ and $(-)$ indicate positively and negatively charged nanoparticles, respectively. 


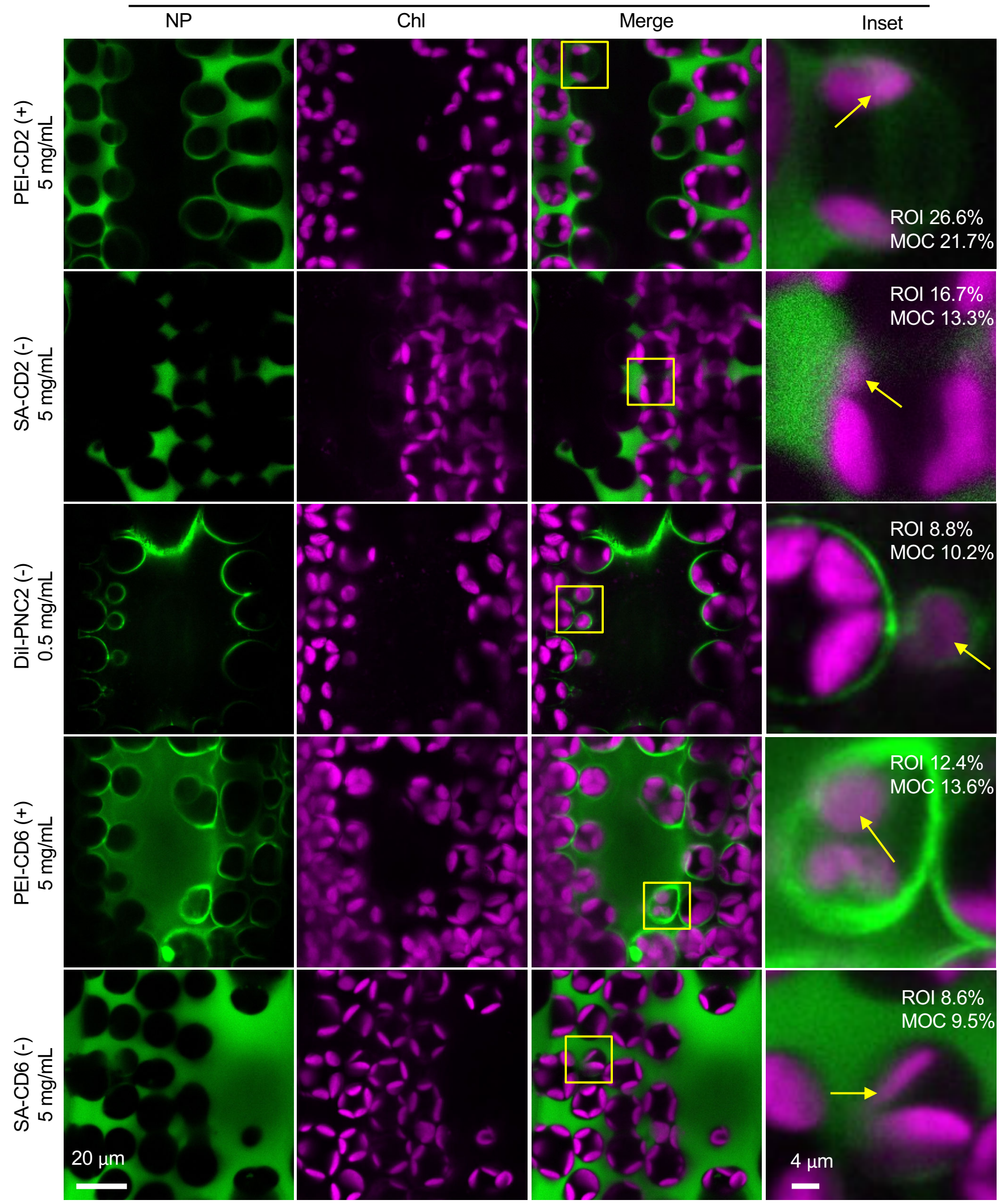

Figure S18. Confocal microscopy images of maize leaf mesophyll cells with chloroplasts after foliar delivery of nanoparticles suspended in Silwet L-77. Inset values are colocalization rates based on ROI method and Manders' overlap coefficients (MOC), representing the fraction of chloroplast pixels overlapping with nanoparticle pixels. Yellow arrows indicate examples of colocalization of nanoparticles (NP, green) and chloroplasts (Chl, magenta), respectively. The (+) and (-) indicate positively and negatively charged nanoparticles, respectively. 


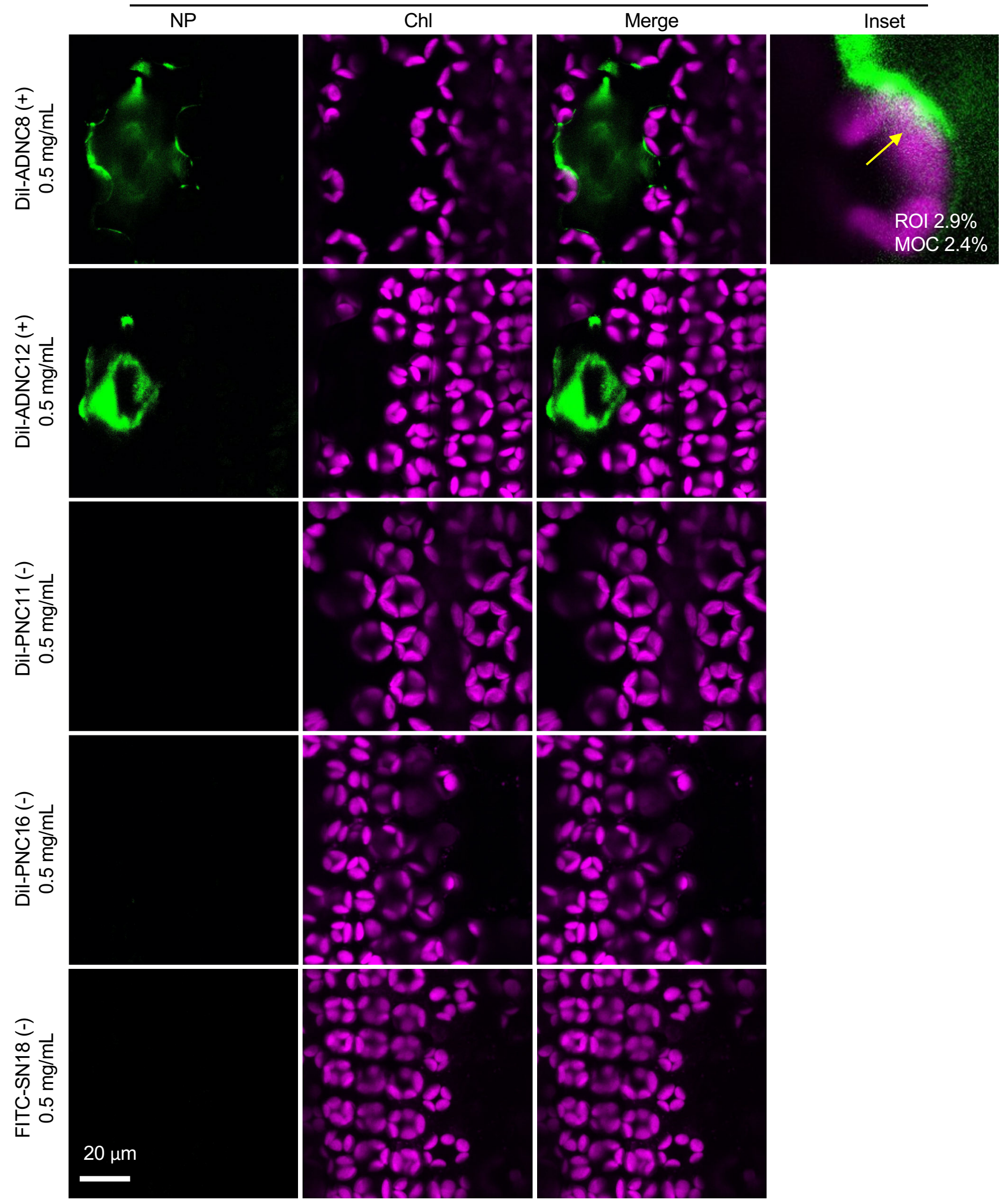

Figure S19. Confocal microscopy images of maize leaf mesophyll cells with chloroplasts after foliar delivery of nanoparticles suspended in Silwet L-77. Inset values are colocalization rates based on ROI method and Manders' overlap coefficients (MOC), representing the fraction of chloroplast pixels overlapping with nanoparticle pixels. Yellow arrows indicate examples of colocalization of nanoparticles (NP, green) and chloroplasts (Chl, magenta), respectively. The $(+)$ and $(-)$ indicate positively and negatively charged nanoparticles, respectively. 

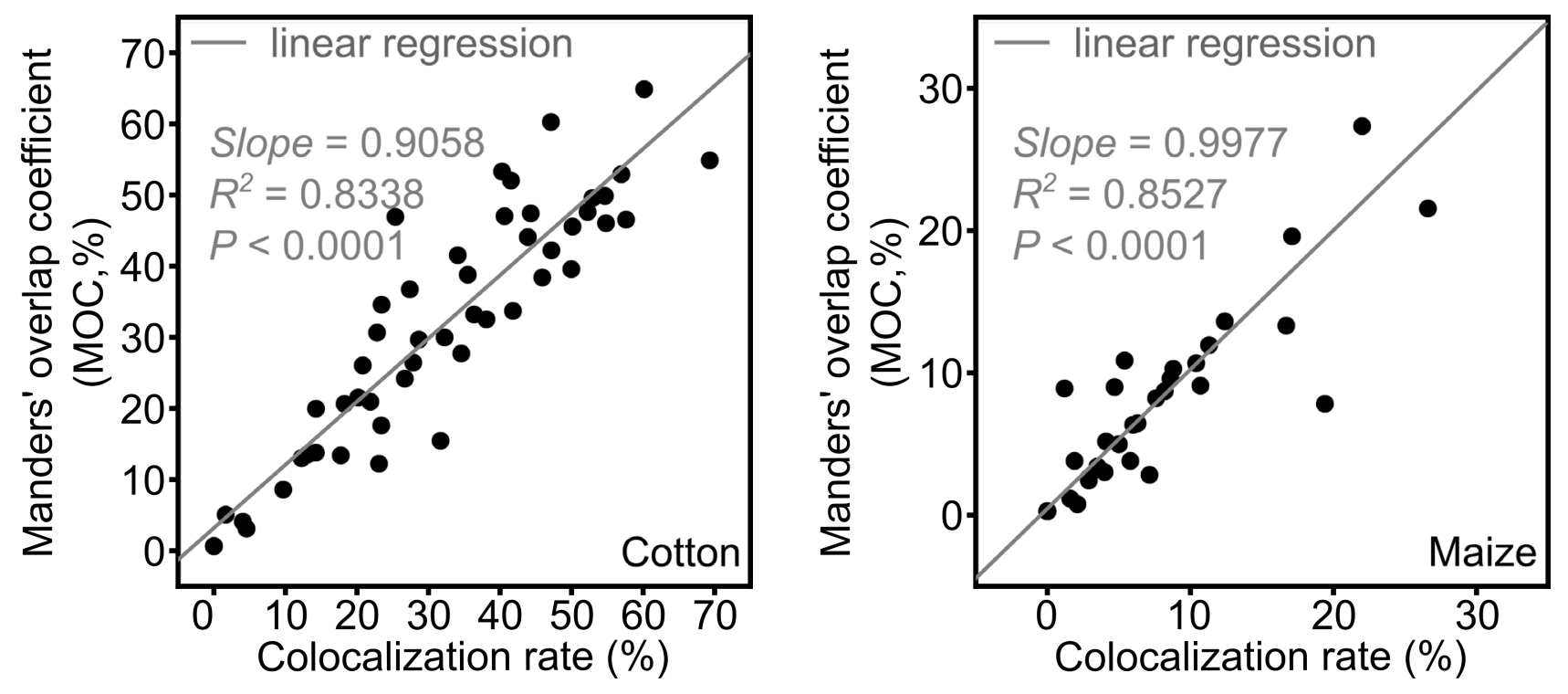

Figure S20. Positive linear correlation between the colocalization rates of chloroplasts based on ROI analysis (percentage of chloroplasts with nanoparticles) and Manders' overlap coefficient (MOC, percentage of chloroplast pixels overlapping with nanoparticle pixels). 
(a)

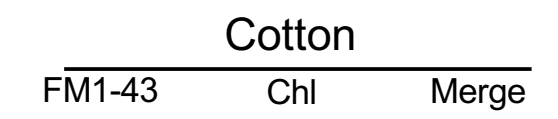

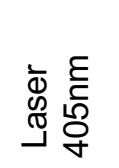
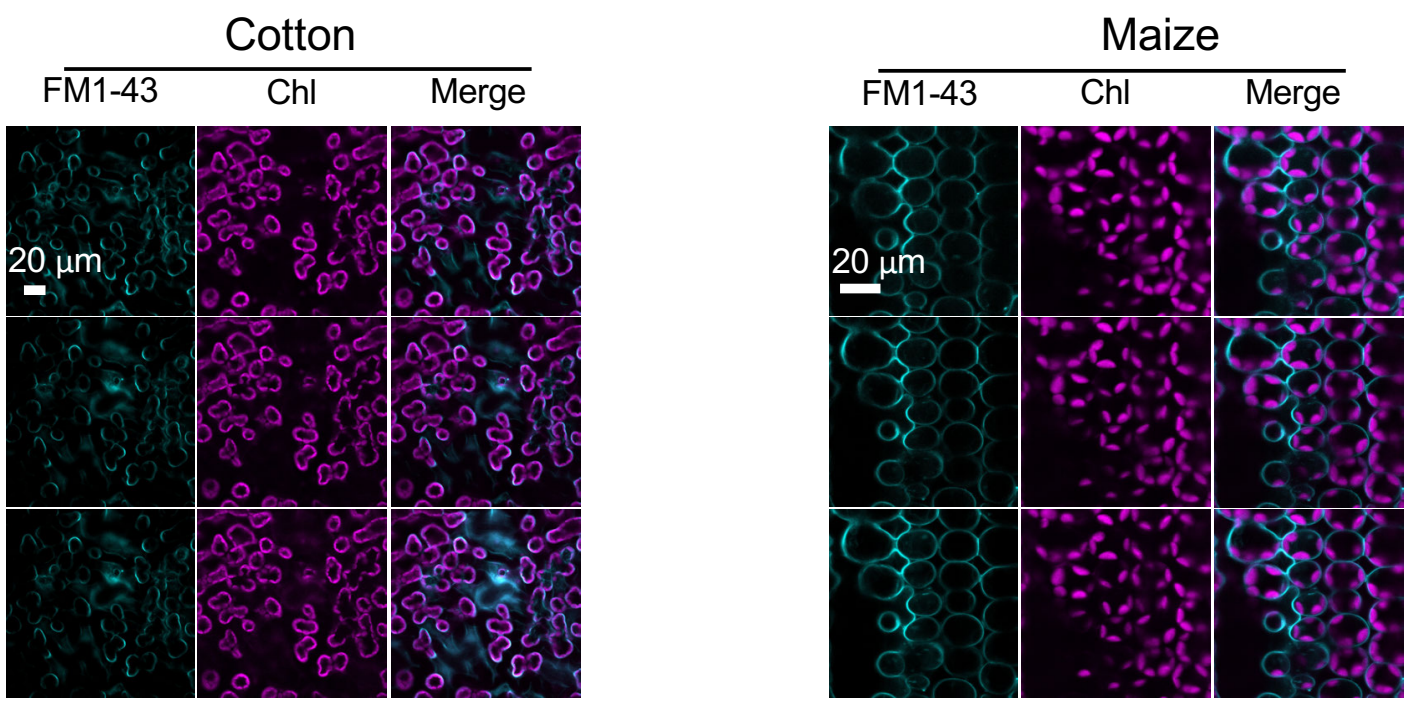

(b)



FM1-43 method

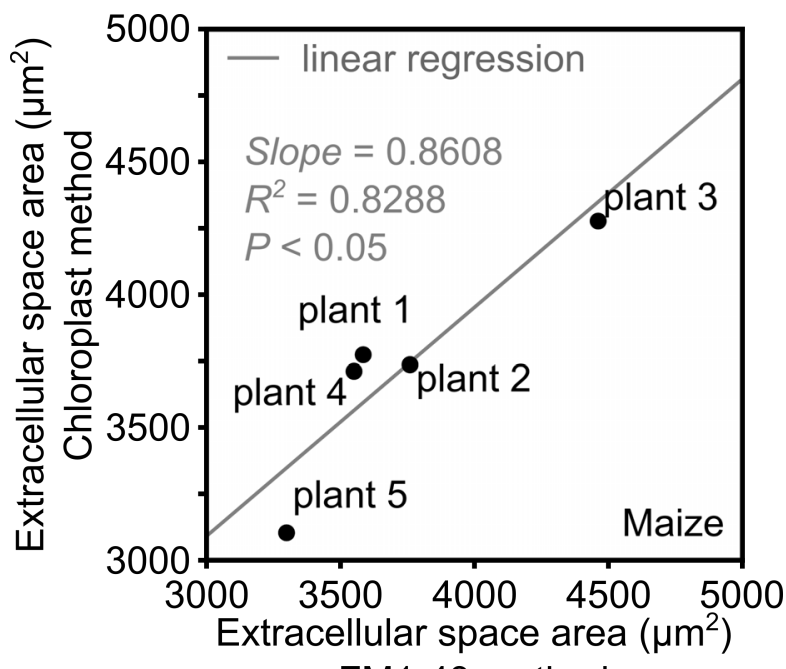

FM1-43 method

Figure S21. a, Representative confocal microscopy images of cotton (left) and maize (right) leaf mesophyll cells with FM1-43 fluorescent dye (10 $\mu \mathrm{g} / \mathrm{mL}, 10 \mathrm{~min}$ ). Laser excitation at 405, 476, and $514 \mathrm{~nm}$. b. Extracellular space area determined by chloroplast autofluorescence arrangement versus FM1-43 labeled cell membranes showing significant and positive correlation. Chl represents chloroplasts (magenta). 


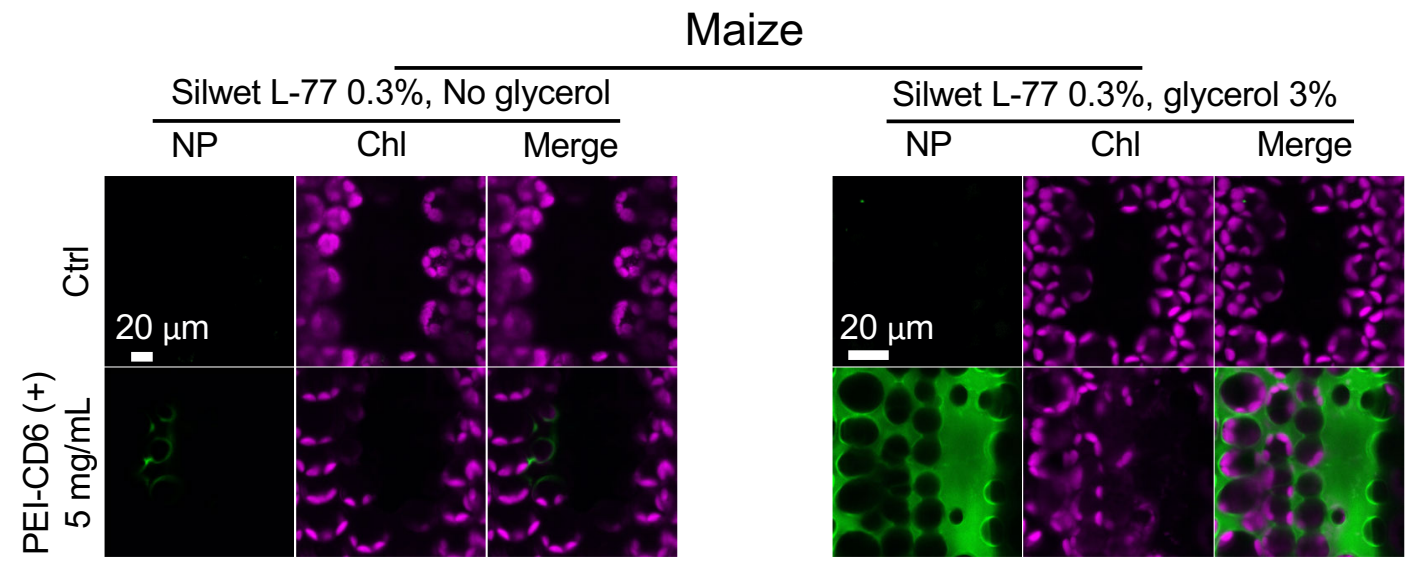

Figure S22. Representative confocal images showing colocalization of chloroplast autofluorescence (Chl, magenta) with foliar delivered nanoparticles (NP, green) suspended in formulations with or without humectant (glycerol, 3\%). NP and $\mathrm{Chl}$ represent nanoparticles (green) and chloroplasts (magenta), respectively. The $(+)$ indicates positively charged nanoparticles. 
(a)

Cotton

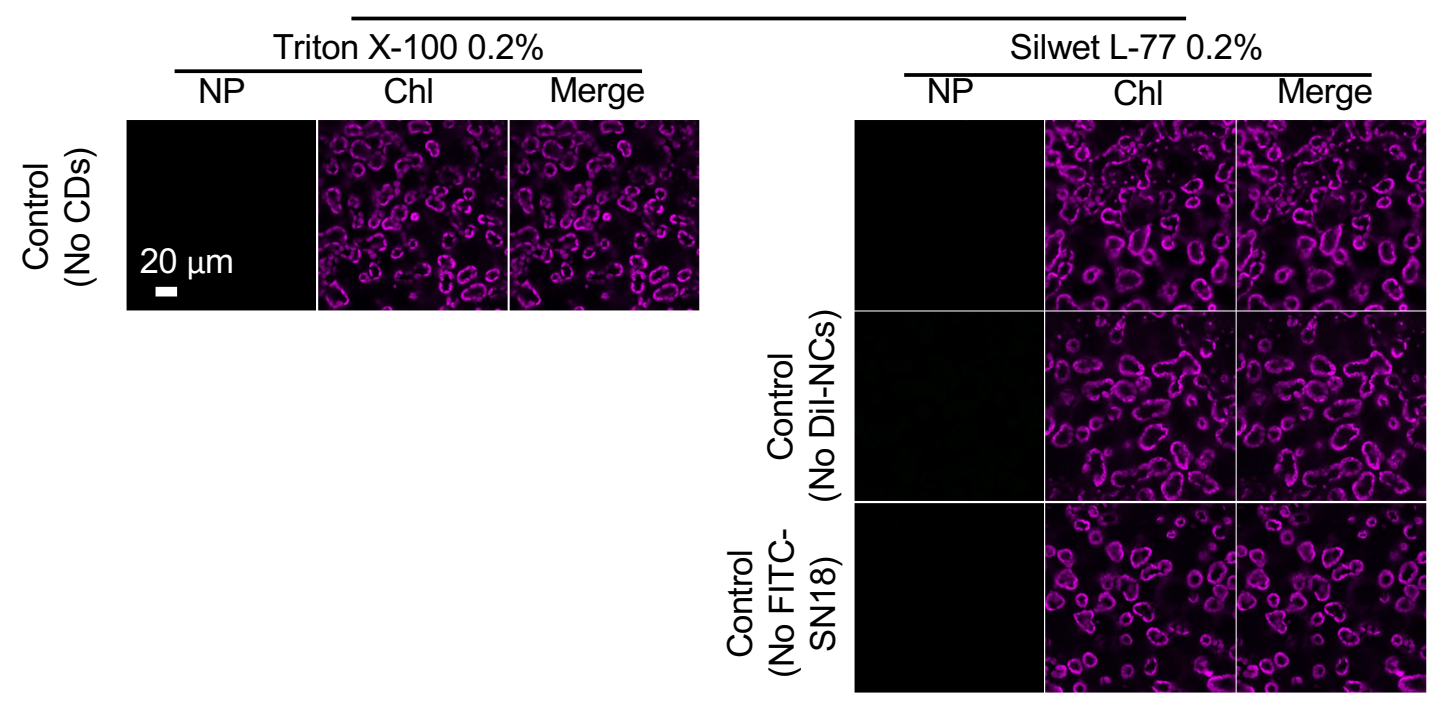

(b)

Maize

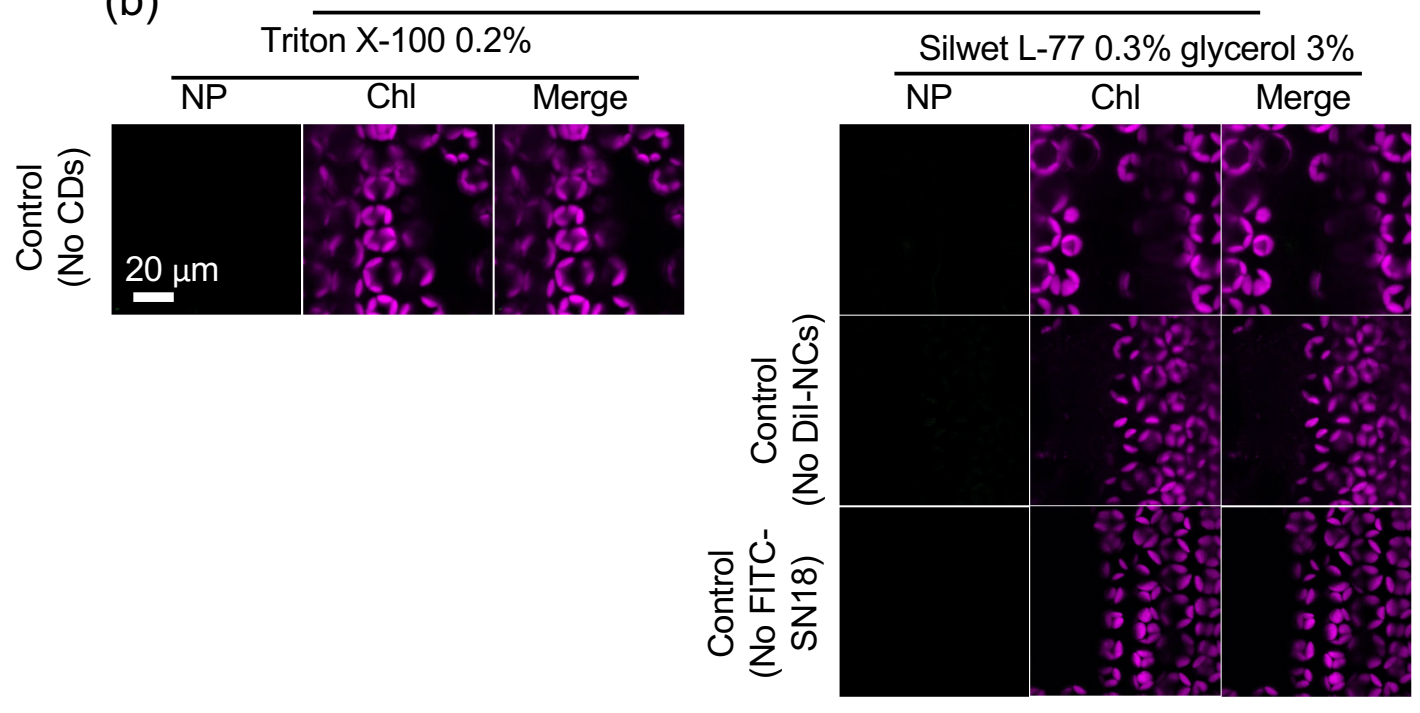

Figure S23. Representative confocal microscopy images showing no fluorescence of nanoparticles (NP) in leaf mesophyll cells when leaves were treated with only control formulations without NPs for $\mathbf{a}$, cotton and b, maize. NP and Chl represent nanoparticles and chloroplasts (magenta), respectively. 
Table S1. Nanoparticle hydrodynamic size and zeta potential averages and corresponding standard deviations used to assess the effect of nanoparticle size and zeta potential on their distribution in plants

\begin{tabular}{|c|c|c|}
\hline Nanoparticles & $\begin{array}{c}\text { Hydrodynamic diameter } \\
\text { (Avg } \pm \text { SD, nm) }\end{array}$ & $\begin{array}{c}\text { Zeta potential } \\
\text { (Avg } \pm \text { SD, mV) }\end{array}$ \\
\hline PEI-CD2 & $1.7 \pm 0.6$ & $+23.3 \pm 1.6$ \\
\hline PEI-CD6 & $5.5 \pm 1.3$ & $+36.6 \pm 2.5$ \\
\hline SA-CD2 & $1.9 \pm 0.6$ & $-13.8 \pm 3.8$ \\
\hline SA-CD6 & $6.4 \pm 1.9$ & $-36.9 \pm 1.8$ \\
\hline Dil-ADNC8 & $7.5 \pm 2.9$ & $+14.9 \pm 0.5$ \\
\hline Dil-ADNC12 & $11.7 \pm 6.1$ & $+34.6 \pm 1.1$ \\
\hline Dil-PNC2 & $1.8 \pm 0.7$ & $-43.5 \pm 1.9$ \\
\hline Dil-PNC11 & $10.8 \pm 8.9$ & $-40.3 \pm 2.5$ \\
\hline Dil-PNC16 & $15.6 \pm 9.0$ & $-52.3 \pm 2.4$ \\
\hline FITC-SN18 & $13.0 \pm 1.8$ & -45.5 \\
\hline
\end{tabular}


Table S2. Efficient nanoparticle delivery regions are defined by $95 \%$ and $90 \%$ confidence ellipses represented by the equation and parameters below. The "+" and "-" symbols indicate positive and negative nanoparticle zeta potential regions, respectively. The $x$ and $y$ axes are hydrodynamic diameter and zeta potential, respectively.

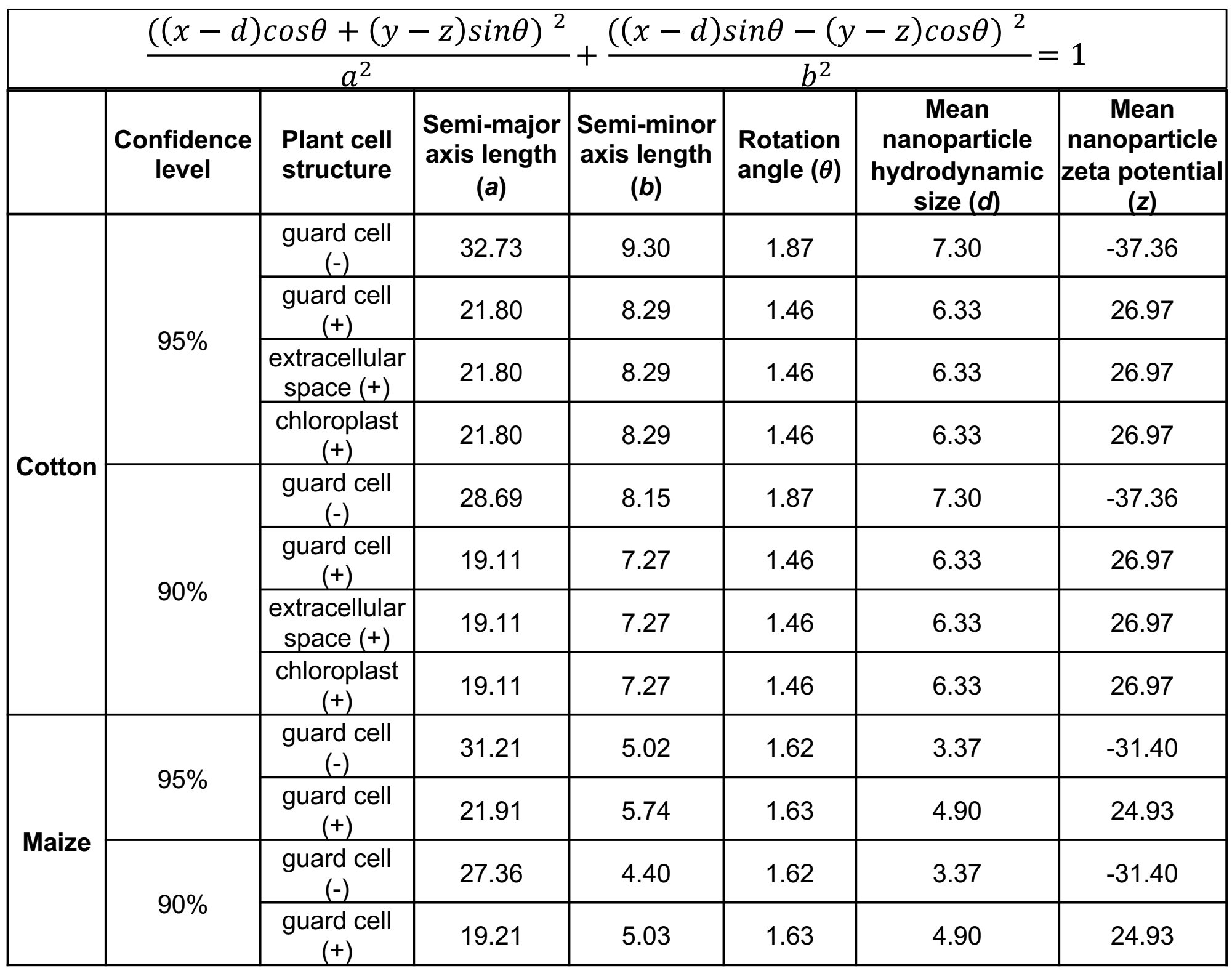

